\title{
POISSON COHOMOLOGY OF HOLOMORPHIC TORIC POISSON MANIFOLDS. I.
}

\author{
WEI HONG
}

\begin{abstract}
A holomorphic toric Poisson manifold is a nonsingular toric variety equipped with a holomorphic Poisson structure, which is invariant under the torus action. In this paper, we computed the Poisson cohomology groups for all holomorphic toric Poisson structures on $\mathbb{C P}^{n}$, with the stand Poisson structure on $\mathbb{C P}^{n}$ as a special case. We also computed the algebraic and the formal Poisson cohomology groups of holomorphic toric Poisson structures on $\mathbb{C}^{n}$.
\end{abstract}

\section{INTRODUCTION}

Holomorphic Poisson manifolds play an important role in modern mathematics. Many interesting works appeared in recently years. The algebraic geometry of the Poisson brackets on projective spaces were studied by Bondal 2 and Polishchuk 23. The close relation of holomorphic Poisson structures with generalized complex geometry and mathematical physics were revealed by Hitchin 12,13 and Gualtieri 11. Deformations of holomorphic Poisson structures appeared in the work of [10, [14 and [16]. In [4, 9], Brown, Goodear and Yakimov studied the standard Poisson structures on affine spaces and flag varieties. Laurent-Gengoux, Stiénon and $\mathrm{Xu} 17$ described the Poisson cohomology of holomorphic Poisson manifolds using Lie algebroids. In various situations, the Poisson cohomology of holomorphic Poisson manifolds were computed 6, 15, 19, 24,

This paper is devoted to the study of the Poisson geometry of toric varieties, especially, the Poisson cohomology of holomorphic toric Poisson manifolds. A holomorphic toric Poisson manifold is a nonsingular toric variety $X$, equipped with a holomorphic Poisson structure $\pi$, which is invariant under the torus action ( Notice that real toric Poisson structures were studied in [5]). Holomorphic toric Poisson manifold is a special case of the " $T$-Poisson manifold" in the sense of [18. In this paper, we computed the Poisson cohomology groups for all holomorphic toric Poisson structures on $X=\mathbb{C P}^{n}$. We also computed the algebraic Poisson cohomology groups and the formal Poisson cohomology groups for all holomorphic toric Poisson structures on $X=\mathbb{C}^{n}$.

This paper is organized in the following way. In Section 2 we give some background knowledge. In Section 3 and Section [4, we study the Poisson cohomology groups of $X=\mathbb{C P}^{n}$. In Section [5 we study the Poisson cohomology groups of $X=\mathbb{C}^{n}$.

The main results of this paper are:

- We give a weight spaces decomposition according to the $\left(\mathbb{C}^{*}\right)^{n}$-representation on the space of holomorphic vector fields and multi-vector fields on $X=\mathbb{C} \mathbf{P}^{n}$ (Theorem 3.4 and Proposition 3.5.).

Key words and phrases. holomorphic Poisson manifolds, Poisson cohomology, toric variety, standard Poisson structure. 
- We described the Poisson cohomology groups of all holomorphic toric Poisson structures on $X=\mathbb{C} \mathbf{P}^{n}$ in Theorem 4.1 and Proposition 4.3, which gives the weight spaces decomposition for the $\left(\mathbb{C}^{*}\right)^{n}$-representation on $H_{\pi}^{\bullet}(X)$.

- We computed the Poisson cohomology groups for the standard Poisson structure on $X=$ $\mathbb{C P}^{n}$ in certain situations. ( Theorem 4.9. Proposition 4.7 and Proposition 4.8)

- For all holomorphic toric Poisson structures on $X=\mathbb{C}^{n}$, we described the corresponding algebraic Poisson cohomology groups and the formal Poisson cohomology groups in Theorem 5.2. which developed Monnier's work in [21].

Acknowledgements I would like to thank Sam Evens, Zhangju Liu, Jianghua Lu, Yannick Voglaire and Ping $\mathrm{Xu}$ for their helpful discussions and comments. Special thanks go to Yat Sun Poon and Bing Zhang for their valuable opinions to the draft of this paper. I wish to express my deep gratitude to Martin Schlichenmaier for his support during the author's stay in Luxembourg. Hong's research was partially supported by NSFC grant 11401441 and FNR grant 5650104.

\section{Preliminary}

\subsection{Poisson cohomology of holomorphic Poisson manifolds.}

Definition 2.1. A holomorphic Poisson manifold is a complex manifold $X$ equipped with a holomorphic bivector field $\pi$ such that $[\pi, \pi]=0$, where $[\cdot, \cdot]$ is the Schouten bracket.

The Poisson cohomology of a holomorphic Poisson manifold is defined in the following way:

Definition 2.2. Let $(X, \pi)$ be a holomorphic Poisson manifold of dimension $\mathrm{n}$. The Poisson cohomology $H_{\pi}^{\bullet}(X)$ is the cohomology group of the complex of sheaves:

$$
\mathcal{O}_{X} \stackrel{d_{\pi}}{\longrightarrow} T_{X} \stackrel{d_{\pi}}{\longrightarrow} \ldots . . \stackrel{d_{\pi}}{\longrightarrow} \wedge^{i-1} T_{X} \stackrel{d_{\pi}}{\longrightarrow} \wedge^{i} T_{X} \stackrel{d_{\pi}}{\longrightarrow} \wedge^{i+1} T_{X} \stackrel{d_{\pi}}{\longrightarrow} \ldots \ldots \stackrel{d_{\pi}}{\longrightarrow} \wedge^{n} T_{X},
$$

where $d_{\pi}=[\pi, \cdot]$.

Lemma 2.3. 17] The Poisson cohomology of a holomorphic Poisson manifold $(X, \pi)$ is isomorphic to the total cohomology of the double complex

$$
\begin{aligned}
& \begin{array}{lll}
\cdots \cdots & \cdots \cdots & \cdots \cdots \\
d_{\pi} \uparrow & d_{\pi} \uparrow & d_{\pi} \uparrow
\end{array}
\end{aligned}
$$

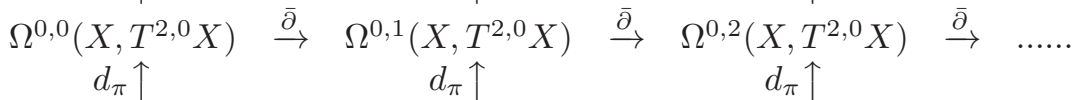

$$
\begin{aligned}
& \Omega^{0,0}\left(X, T^{1,0} X\right) \quad \stackrel{\bar{\partial}}{\rightarrow} \quad \Omega^{0,1}\left(X, T^{1,0} X\right) \quad \stackrel{\bar{\partial}}{\rightarrow} \quad \Omega^{0,2}\left(X, T^{1,0} X\right) \stackrel{\bar{\partial}}{\rightarrow} \quad \ldots \ldots \\
& d_{\pi} \uparrow \quad d_{\pi} \uparrow \quad d_{\pi} \uparrow \\
& \Omega^{0,0}\left(X, T^{0,0} X\right) \stackrel{\bar{\partial}}{\rightarrow} \Omega^{0,1}\left(X, T^{0,0} X\right) \stackrel{\bar{\partial}}{\rightarrow} \quad \Omega^{0,2}\left(X, T^{0,0} X\right) \stackrel{\bar{\partial}}{\rightarrow} \ldots \ldots
\end{aligned}
$$

Lemma 2.4. Let $(X, \pi)$ be a holomorphic Poisson manifold. If all the higher cohomology groups $H^{i}\left(X, \wedge^{j} T_{X}\right)$ vanish for $i>0$, then the Poisson cohomology $H_{\pi}^{\bullet}(X)$ is isomorphic to the cohomology of the complex

$$
H^{0}\left(X, \mathcal{O}_{X}\right) \stackrel{d_{\pi}}{\longrightarrow} H^{0}\left(X, T_{X}\right) \stackrel{d_{\pi}}{\longrightarrow} H^{0}\left(X, \wedge^{2} T_{X}\right) \stackrel{d_{\pi}}{\longrightarrow} \ldots \stackrel{d_{\pi}}{\longrightarrow} H^{0}\left(X, \wedge^{n} T_{X}\right),
$$

where $d_{\pi}=[\pi, \cdot]$. 
2.2. Toric varieties. In this section, we recall some classical knowledge of toric varieties. One may consult [7, 8] and [22].

Definition 2.5. A toric variety is an irreducible variety $X$ such that

(1) $\left(\mathbb{C}^{*}\right)^{n}$ is a Zariski open set of $X$, and

(2) the action of $\left(\mathbb{C}^{*}\right)^{n}$ on itself extends to an action of $\left(\mathbb{C}^{*}\right)^{n}$ on $X$.

Toric varieties can also be described by a Lattice $N \cong \mathbb{Z}^{n}$ and a fan $\Delta$ in $N_{\mathbb{R}}=N \otimes_{\mathbb{Z}} \mathbb{R} \cong \mathbb{R}^{n}$.

Let $M=\operatorname{Hom}_{\mathbb{Z}}(N, \mathbb{Z})$ and $T_{N}=H \operatorname{Hom}_{\mathbb{Z}}\left(M, \mathbb{C}^{*}\right)=N \otimes_{\mathbb{Z}} \mathbb{C}^{*}$. Then $T_{N} \cong\left(\mathbb{C}^{*}\right)^{n}$. Moreover, we have $M \cong \operatorname{Hom}\left(T_{N}, \mathbb{C}^{*}\right)$ and $N \cong \operatorname{Hom}\left(\mathbb{C}^{*}, T_{N}\right)$.

Each element $m$ in $M$ gives rise to a character $\chi^{m} \in \operatorname{Hom}\left(T_{N}, \mathbb{C}^{*}\right)$, given by

$$
\chi^{m}(t)=\langle t, m\rangle \quad \text { for } \quad t \in T_{N} .
$$

Each element $a$ in $N$ gives rise to a one-parameter subgroup $\gamma_{a} \in H o m\left(\mathbb{C}^{*}, T_{N}\right)$ given by

$$
\gamma_{a}(\lambda)(m)=\lambda^{\langle a, m\rangle} \text { for } \lambda \in \mathbb{C}^{*} \text { and } m \in M \text {. }
$$

Fix a $\mathbb{Z}$-basis $\left\{e_{1}, \ldots e_{n}\right\}$ of $N$ and let $\left\{e_{1}^{*}, \ldots e_{n}^{*}\right\}$ be the dual basis of $M$. Let $t_{i}=\left\langle t, e_{i}^{*}\right\rangle(1 \leq i \leq n)$. Then there is an isomorphism

$$
T_{N} \cong\left(\mathbb{C}^{*}\right)^{n}: t \longleftrightarrow\left(t_{1}, t_{2}, \ldots t_{n}\right),
$$

where $t_{1}, t_{2}, \ldots t_{n} \in \mathbb{C}^{*}$ are considered as the coordinates on $T_{N}$.

For $m=\sum_{i=1}^{n} m_{i} e_{i}^{*}$, we have $\chi^{m}=t_{1}^{m_{1}} t_{2}^{m_{2}} \ldots t_{n}^{m_{n}}$, which is a Laurent monomial on $T_{N}$. For $a=\sum_{i=1}^{n} a_{i} e_{i}$, the one-parameter subgroup $\gamma_{a}$ can be written as $\gamma_{a}(\lambda)=\left(\lambda^{a_{1}}, \ldots \lambda^{a_{n}}\right)$ for $\lambda \in \mathbb{C}^{*}$.

Definition 2.6. A subset $\sigma$ of $N_{\mathbb{R}}$ is called a rational polyhedral cone (with apex at the origin $O$ ), if there there exist a finite number of elements $e_{1}, e_{2}, \ldots, e_{s}$ in $N$ such that

$$
\begin{aligned}
\sigma & =\mathbb{R}_{\geq 0} e_{1}+\ldots \mathbb{R}_{\geq 0} e_{s} \\
& =\left\{a_{1} e_{1}+\ldots+a_{s} e_{s} \mid a_{i} \in \mathbb{R}, a_{i} \geq 0 \text { for all } 0 \leq i \leq s\right\},
\end{aligned}
$$

where we denote by $R_{\geq 0}$ the set of nonnegative real numbers.

(1) $\sigma$ is strongly convex if $\sigma \cap(-\sigma)=O$.

(2) The dimension of $\sigma$ is the dimension of the smallest subspace of $N_{\mathbb{R}}$ containing $\sigma$.

In this paper, a cone is always a rational polyhedral cone.

Let $M_{\mathbb{R}}=M \otimes_{Z} \mathbb{R}$. The canonical $\mathbb{Z}$-bilinear pairing

$$
\langle,\rangle: M \times N \rightarrow \mathbb{Z}
$$

extends to a $\mathbb{R}$-bilinear pairing $\langle\rangle:, M_{\mathbb{R}} \times N_{\mathbb{R}} \rightarrow \mathbb{R}$. Given a cone $\sigma \in N_{\mathbb{R}}$, its dual cone in $M_{\mathbb{R}}$ is defined to be

$$
\sigma^{\vee}=\left\{x \in M_{\mathbb{R}} \mid\langle x, y\rangle \geq 0 \text { for all } y \in \sigma\right\} .
$$

A face of $\sigma$ is a subset of $\sigma$, which can be written as $m^{\perp} \cap \sigma=\{x \in \sigma \mid\langle x, m\rangle=0\}$ for $m \in \sigma^{\vee}$.

Definition 2.7. A fan in $N$ is a nonempty collection $\Delta$ of strongly convex rational polyhedral cones in $N_{\mathbb{R}}$ satisfying the following conditions:

(1) Every face of any $\sigma \in \Delta$ is contained in $\Delta$.

(2) For any $\sigma, \sigma^{\prime} \in \Delta$, the intersection $\sigma \cap \sigma^{\prime}$ is a face of both $\sigma$ and $\sigma^{\prime}$. 
The union $|\Delta|=\cup_{\sigma \in \Delta} \sigma$ is called the support of $\Delta$.

In this paper, we assume that fans are finite, i.e., a fan consist of only finite number of cones.

Given a fan $\Delta$, the set of $k$-dimensional cones in $\Delta$ is denoted by $\Delta(k)(0 \leq k \leq n)$. The primitive element of $\alpha \in \Delta(1)$ is the unique generator of $\alpha \cap N$, denoted by $e(\alpha)$.

Let $S_{\sigma}=\sigma^{\vee} \cap M$. For a strongly convex rational polyhedral cone $\sigma$ in $N_{\mathbb{R}}$, the semigroup algebra

$$
\mathbb{C}\left[S_{\sigma}\right]=\bigoplus_{m \in S_{\sigma}} \mathbb{C} \chi^{m}
$$

is a finitely generated commutative $\mathbb{C}$-algebra. The affine variety $U_{\sigma}=\operatorname{Spec}\left(\mathbb{C}\left[S_{\sigma}\right]\right)$ is a $n$ dimensional toric variety.

Theorem 2.8. Given a lattice $N \cong \mathbb{Z}^{n}$ and a fan $\Delta$ in $N_{\mathbb{R}} \cong R^{n}$, there exists a toric variety $X_{\Delta}$, obtained from the affine variety $U_{\sigma}, \sigma \in \Delta$, by gluing together $U_{\sigma}$ and $U_{\tau}$ along their common open subset $U_{\sigma \cap \tau}$ for all $\sigma, \tau \in \Delta$.

A cone $\sigma$ is called nonsingular if $\sigma$ can be written as

$$
\sigma=\mathbb{R}_{\geq 0} e_{1}+\ldots \mathbb{R}_{\geq 0} e_{s}
$$

where $\left\{e_{1}, e_{2}, \ldots, e_{s}\right\}$ is a subset of a $\mathbb{Z}$-basis of $N$.

Theorem 2.9. Let $X_{\Delta}$ be the toric variety associated with a fan $\Delta$ in $N_{\mathbb{R}}$. Then

(1) $X_{\Delta}$ is compact $\Longleftrightarrow|\Delta|=N_{\mathbb{R}}$.

(2) $X_{\Delta}$ is nonsingular $\Longleftrightarrow$ each $\sigma \in \Delta$ is nonsingular.

For a nonsingular toric variety $X_{\Delta}$, the action map $T_{N} \times X_{\Delta} \rightarrow X_{\Delta}$ is a holomorphic map. Let $N_{\mathbb{C}}=N \otimes_{\mathbb{Z}} \mathbb{C}$. Then $\operatorname{Lie}\left(T_{N}\right) \cong N_{\mathbb{C}}$. By identification of $\operatorname{Lie}\left(T_{N}\right)$ with $N_{\mathbb{C}}$, the infinitesimal action of Lie algebra $\operatorname{Lie}\left(T_{N}\right)$ on $X_{\Delta}$ defines a map

$$
\rho: N_{\mathbb{C}}=N \otimes_{\mathbb{Z}} \mathbb{C} \rightarrow \mathfrak{X}\left(X_{\Delta}\right) .
$$

The image of $\rho$ are holomorphic vector fields on $X_{\Delta}$. For any $e \in N_{\mathbb{C}}$ and $m \in M$, we have

$$
\rho(e)\left(\chi^{m}\right)=\langle e, m\rangle \chi^{m}
$$

where $\chi^{m}$ is considered as a rational function on $X_{\Delta}$. By abuse of notation, we denote the induced map

$$
\wedge^{k} N_{\mathbb{C}} \rightarrow \mathfrak{X}^{k}\left(X_{\Delta}\right)
$$

also by $\rho$.

Example 2.10. Let $X=\mathbb{C}^{n}$ and $\left(z_{1}, \ldots, z_{n}\right)$ be the standard coordinates on it. There is a nature embedding $\left(\mathbb{C}^{*}\right)^{n} \hookrightarrow \mathbb{C}^{n}$. The $\left(\mathbb{C}^{*}\right)^{n}$-action on $X=\mathbb{C}^{n}$ defined by

$$
\left(t_{1}, t_{2}, \ldots, t_{n}\right) \cdot\left(z_{1}, z_{2}, \ldots, z_{n}\right)=\left(t_{1} z_{1}, t_{2} z_{2}, \ldots, t_{n} z_{n}\right)
$$

makes $X=\mathbb{C}^{n}$ a toric variety.

Let $\left\{e_{1}=(1,0,, . ., 0), \ldots, e_{n}=(0, \ldots, 0,1)\right\}$ be the standard $\mathbb{Z}$-basis of $N=\mathbb{Z}^{n}$. Let

$$
\sigma=\sum_{i=1}^{n} \mathbb{R}_{\geq 0} e_{i}
$$


Let the fan $\Delta$ be the collection of the cones of the following form:

$$
\sum_{s=1}^{k} \mathbb{R}_{\geq 0} e_{i_{s}}, \quad\left\{i_{1}, i_{2}, \ldots, i_{k}\right\} \subseteq\{1, \ldots, n\} .
$$

Then we have $X_{\Delta}=U_{\sigma} \cong \mathbb{C}^{n}$.

Let $\left\{e_{1}^{*}, e_{2}^{*}, \ldots, e_{n}^{*}\right\}$ be the dual basis of $\left\{e_{1}, e_{2}, \ldots, e_{n}\right\}$ in $M$. Then we have

$$
\chi^{e_{i}^{*}}=z_{i}, \quad 1 \leq i \leq n .
$$

For $m=\sum_{i=1}^{n} m_{i} e_{i}^{*}$, the rational function $\chi^{m}$ on $X_{\Delta}=\mathbb{C}^{n}$ can be written as

$$
\chi^{m}=z_{1}^{m_{1}} \ldots z_{n}^{m_{n}} .
$$

Example 2.11. Let $X=\mathbb{C P}^{n}$ and $\left[z_{0}, z_{1}, \ldots, z_{n}\right]$ be homogenous coordinates on it. The map

$$
\left(\mathbb{C}^{*}\right)^{n} \rightarrow \mathbb{C} \mathbf{P}^{n}
$$

defined by $\left(t_{1}, t_{2}, \ldots, t_{n}\right) \mapsto\left[1, t_{1}, t_{2}, \ldots, t_{n}\right]$ allows us to identify $\left(\mathbb{C}^{*}\right)^{n}$ with the Zariski open subset $\left\{\left[z_{0}, z_{1}, \ldots, z_{n}\right] \in \mathbb{C} \mathbf{P}^{n} \mid z_{i} \neq 0,0 \leq i \leq n\right\}$ of $\mathbb{C} \mathbf{P}^{n}$. The $\left(\mathbb{C}^{*}\right)^{n}$ action on $\mathbb{C} \mathbf{P}^{n}$ given by

$$
\left(t_{1}, \ldots, t_{n}\right) \cdot\left[z_{0}, z_{1}, \ldots, z_{n}\right]=\left[z_{0}, t_{1} z_{1}, \ldots, t_{n} z_{n}\right]
$$

makes $X=\mathbb{C} \mathbf{P}^{n}$ to be a toric variety.

Let $e_{0}=(-1,-1, \ldots,-1), e_{1}=(1,0,, . ., 0), \ldots, e_{n}=(0, \ldots, 0,1)$ be vectors in $N=\mathbb{Z}^{n}$. Let the fan $\Delta$ be the collection of the cones of the following form:

$$
\sigma=\sum_{s=1}^{k} \mathbb{R}_{\geq 0} e_{i_{s}}, \quad\left\{i_{1}, i_{2}, \ldots, i_{k}\right\} \subsetneq\{0,1, \ldots, n\} .
$$

Then we have $X_{\Delta} \cong \mathbb{C} \mathbf{P}^{n}$. Let

$$
\sigma_{i}=\sum_{s=1}^{n} \mathbb{R}_{\geq 0} e_{i_{s}}, \quad\left\{i_{1}, i_{2}, \ldots, i_{n}\right\}=\{0,1, \ldots, n\} \backslash\{i\} .
$$

Then $U_{\sigma_{i}}$ can be identified with the affine open set $U_{i}=\left\{\left[z_{0}, z_{1}, \ldots, z_{n}\right] \in \mathbb{C P}^{n} \mid z_{i} \neq 0\right\}$.

Choose the $\mathbb{Z}$-basis $\left\{e_{1}, e_{2}, \ldots, e_{n}\right\}$ of $N$ and its dual basis $\left\{e_{1}^{*}, e_{2}^{*}, \ldots, e_{n}^{*}\right\}$ of $M$. Then $t_{i}=\chi^{e_{i}^{*}}$ $(1 \leq i \leq n)$ can be considered as the affine coordinates on $U_{0}$, i.e., $t_{i}=\frac{z_{i}}{z_{0}}$. And for $m=\sum_{i=1}^{n} m_{i} e_{i}^{*}$, the rational function $\chi^{m}$ on $X_{\Delta}=\mathbb{C} \mathbf{P}^{n}$ can be written as

$$
\chi^{m}=t_{1}^{m_{1}} t_{2}^{m_{2}} \ldots t_{n}^{m_{n}}=z_{0}^{m_{0}} z_{1}^{m_{1}} \ldots z_{n}^{m_{n}}
$$

where $m_{0}=-\sum_{i=1}^{n} m_{i}$.

\subsection{Holomorphic toric Poisson structures.}

Definition 2.12. Let $X$ be a nonsingular toric variety. If a holomorphic Poisson structure $\pi$ on $X$ is invariant under the torus action, then $\pi$ is called a holomorphic toric Poisson structure on $X$, and $X$ is called a holomorphic toric Poisson manifold.

Proposition 2.13. Let $X_{\Delta}$ be a nonsingular toric variety associated with a fan $\Delta$ in $N_{\mathbb{R}}$. Then the set of holomorphic toric Poisson structures on $X$ coincide with $\rho\left(\wedge^{2} N_{\mathbb{C}}\right)$, where $\rho: \wedge^{2} N_{\mathbb{C}} \rightarrow \mathfrak{X}^{2}\left(X_{\Delta}\right)$ is defined in (2.5).

Proposition 2.13 can be state in an equivalent way: 
Proposition 2.14. Let $X_{\Delta}$ be a nonsingular toric variety associated with a fan $\Delta$ in $N_{\mathbb{R}}$. Suppose that $\left\{e_{1}, e_{2}, \ldots, e_{n}\right\}$ is a basis of $N \subset N_{\mathbb{C}}$. Let $v_{i}=\rho\left(e_{i}\right)(1 \leq i \leq n)$ be holomorphic vector fields on $X_{\Delta}$, where $\rho: N_{\mathbb{C}} \rightarrow \mathfrak{X}\left(X_{\Delta}\right)$ is defined in (2.3). Then $\pi$ is a holomorphic toric Poisson structure on $X_{\Delta}$ if and only if $\pi$ can be written as

$$
\pi=\sum_{1 \leq i<j \leq n} a_{i j} v_{i} \wedge v_{j}
$$

where $a_{i j}(1 \leq i<j \leq n)$ are complex constants.

Proof. $\quad \bullet \Leftarrow$ : Suppose that

$$
\pi=\sum_{1 \leq i<j \leq n} a_{i j} v_{i} \wedge v_{j}
$$

where $a_{i j}(1 \leq i<j \leq n)$ are complex constants. As $T_{N}$ is abelian, we have that $\left[v_{i}, v_{j}\right]=0$ for all $1 \leq i<j \leq n$, which imply $[\pi, \pi]=0$. Obviously, $\pi$ is holomorphic and $T_{N}$-invariant. Thus $\pi=\sum_{1<i<j \leq n} a_{i j} v_{i} \wedge v_{j}$ is a holomorphic toric Poisson structure on $X_{\Delta}$.

- $\Rightarrow$ : Suppose that $\pi$ is a holomorphic toric Poisson structure on $X_{\Delta}$. Then the restriction of $\pi$ on $T_{N} \subset X_{\Delta}$ is a holomorphic toric Poisson structure on $T_{N}$. We denoted it by $\tilde{\pi}$. Any $T_{N}$-invariant holomorphic bi-vector field on $T_{N} \subset X_{\Delta}$ can be written as

$$
\sum_{1 \leq i<j \leq n} a_{i j} \tilde{v}_{i} \wedge \tilde{v}_{j}
$$

where $a_{i j}(1 \leq i<j \leq n)$ are complex constants, and $\tilde{v}_{i}(1 \leq i \leq n)$ are the restriction of the vector fields $v_{i}(1 \leq i \leq n)$ on $T_{N}$. Hence $\tilde{\pi}$ can be written as

$$
\tilde{\pi}=\sum_{1 \leq i<j \leq n} a_{i j} \tilde{v}_{i} \wedge \tilde{v}_{j} .
$$

As $T_{N}$ is a dense open set of $X_{\Delta}$, we have

$$
\pi=\sum_{1 \leq i<j \leq n} a_{i j} v_{i} \wedge v_{j} .
$$

Example 2.15. Let $X=\mathbb{C}^{n}$ and $\left(z_{1}, \ldots, z_{n}\right)$ be the standard coordinates on it. As we have shown in Example 2.10, $X=\mathbb{C}^{n}$ is a toric variety. Let $v_{i}=\rho\left(e_{i}\right)(1 \leq i \leq n)$. Then we have

$$
v_{i}=z_{i} \frac{\partial}{\partial z_{i}} .
$$

Any holomorphic toric Poisson structures on $X=\mathbb{C}^{n}$ can be written as

$$
\pi=\sum_{1 \leq i<j \leq n} a_{i j} v_{i} \wedge v_{j},
$$

where $a_{i j}(1 \leq i<j \leq n$ are complex constants.

Example 2.16. Let $X=\mathbb{C} \mathbf{P}^{n}$ and let $\left[z_{0}, z_{1}, \ldots, z_{n}\right]$ be homogenous coordinates on it. As we have shown in Example 2.11 $X=\mathbb{C P}^{n}$ is a toric variety. Let $\mathcal{P}=\mathbb{C}^{n+1} \backslash\{0\}=\left\{\left(z_{0}, z_{1}, \ldots, z_{n}\right) \mid\right.$ $z_{0}, z_{1}, \ldots, z_{n}$ are not all zeros $\}$, and let $p: \mathcal{P}=\mathbb{C}^{n+1} \backslash\{0\} \rightarrow \mathbb{C P}^{n}$ be the canonical projection. 
Then $v_{i}=p_{*}\left(z_{i} \frac{\partial}{\partial z_{i}}\right)(0 \leq i \leq n)$ are holomorphic vector fields on $X$, and $\sum_{i=0}^{n} v_{i}=0$. Moreover, by Equation (2.4) and Equation (2.7), we have

$$
v_{i}=\rho\left(e_{i}\right) \quad \text { for } \quad i=0,1, \ldots, n .
$$

Thus any holomorphic toric Poisson structures on $X$ can be written as

$$
\pi=\sum_{1 \leq i<j \leq n} a_{i j} v_{i} \wedge v_{j}
$$

where $a_{i j}(1 \leq i<j \leq n$ are complex constants.

Given a holomorphic toric Poisson manifold $(X, \pi)$, as the torus action on $X$ is holomorphic and $\pi$ is invariant under the torus action, we have the following lemma.

Lemma 2.17. For a holomorphic toric Poisson manifold $(X, \pi)$, the torus action on $X$ induces a torus action on the Poisson cohomology groups $H_{\pi}^{\bullet}(X)$.

2.4. The standard Poisson structure on $\mathbb{C P}^{n}$. In [4,9, Brown, Goodear and Yakimov studied the geometry of the standard Poisson structures on affine spaces and flag varieties. Let us review the definition of the standard Poisson structure on flag varieties.

Let $G$ be a connected complex reductive algebraic group with maximal torus $H$. Denote the corresponding Lie algebra by $\mathfrak{g}$ and $\mathfrak{h}$. Denote $\Delta_{+}\left(\Delta_{-}\right)$the set of all positive (negative) roots of $\mathfrak{g}$ with respect to $\mathfrak{h}$.

The standard $r$-matrix of $\mathfrak{g}$ is given by

$$
r_{\mathfrak{g}}=\sum_{\alpha \in \Delta_{+}} e_{\alpha} \wedge e_{-\alpha}
$$

where $e_{\alpha}$ and $e_{-\alpha}$ are root vectors of $\alpha$ and $-\alpha$, normalized by $\left\langle e_{\alpha}, f_{\alpha}\right\rangle=1$. The standard Poisson structure on $G$ is given by

$$
\pi_{G}=L\left(r_{\mathfrak{g}}\right)-R\left(r_{\mathfrak{g}}\right),
$$

where $L\left(r_{\mathfrak{g}}\right)$ and $R\left(r_{\mathfrak{g}}\right)$ refer to the left and right invariant bi-vector fields on $G$ associated to $r_{\mathfrak{g}} \in \wedge^{2} \mathfrak{g} \cong \wedge^{2} T_{e} G$.

For a parabolic group $P$ containing $H, X=G / P$ is a flag variety. The action of $G$ on $X=G / P$ induces a map $\mu: \mathfrak{g} \rightarrow \mathfrak{X}(X)$. By abuse of notations, the induced maps $\wedge^{k} \mathfrak{g} \rightarrow \mathfrak{X}^{k}(X)$ are also denoted by $\mu$. The natural projection

$$
\phi: G \rightarrow X=G / P
$$

induces the following Poisson structure on the flag variety $X=G / P$ :

$$
\pi_{s t}=\phi_{*}\left(\pi_{G}\right)=\mu\left(r_{\mathfrak{g}}\right),
$$

called the standard Poisson structure on the flag varieties. The standard Poisson structure $\pi_{s t}$ is a holomorphic Poisson structure on the flag variety $G / P$.

Next we will focus on the standard Poisson structure on $\mathbb{C} \mathbf{P}^{n}$.

Let $G=G L(n+1, \mathbb{C})$. Let $H$ be consisting of the diagonal matrices in $G L(n+1, \mathbb{C})$ and $P$ consisting of matrices of the following form $\left(\begin{array}{ll}\lambda & b \\ 0 & D\end{array}\right)$, where $\lambda \in \mathbb{C}^{*}, b \in \mathbb{C}^{n}, D \in G L(n, \mathbb{C})$. Then $X=G / P$ becomes the projective space $\mathbb{C} \mathbf{P}^{n}$. 
The left action of $G L(n+1, \mathbb{C})$ on $X=\mathbb{C P}^{n}$ can be written as:

$$
A \cdot\left[z_{0}, z_{1}, \ldots, z_{n}\right] \mapsto p\left(\left(z_{0}, z_{1}, \ldots, z_{n}\right) A^{t}\right),
$$

where $A \in G L(n+1, \mathbb{C}),\left[z_{0}, z_{1}, \ldots, z_{n}\right] \in \mathbb{C P}^{n},\left(z_{0}, z_{1}, \ldots, z_{n}\right) \in \mathbb{C}^{n+1}$, and $p$ is the canonical projection

$$
\mathbb{C}^{n+1} \backslash\{0\} \stackrel{p}{\rightarrow} \mathbb{C P}^{n}:\left(z_{0}, z_{1}, \ldots, z_{n}\right) \rightarrow\left[z_{0}, z_{1}, \ldots, z_{n}\right] .
$$

The standard $r$-matrix of $\mathfrak{g}=g l(n+1, \mathbb{C})$ can be written as

$$
r_{\mathfrak{g}}=\sum_{0 \leq i<j \leq n} e_{i j} \wedge e_{j i}
$$

where $e_{i j}$ denotes the matrix having 1 in the $(i+1, j+1)$ position and 0 elsewhere.

Now we are ready to compute the standard Poisson structure on $\mathbb{C} \mathbf{P}^{n}$.

Proposition 2.18. Let $X=\mathbb{C P}^{n}=G L(n+1, \mathbb{C}) / P$. Let $v_{i}=p_{*}\left(z_{i} \frac{\partial}{\partial z_{i}}\right)(i=0,1, \ldots, n)$. Then the standard Poisson structure on $X=\mathbb{C P}^{n}$ can be written as

$$
\pi_{s t}=\sum_{1 \leq i<j \leq n} v_{i} \wedge v_{j}
$$

Proof. By computation, we have

$$
\mu\left(e_{i j}\right)=p_{*}\left(z_{j} \frac{\partial}{\partial z_{i}}\right), \quad \text { and } \quad \mu\left(e_{j i}\right)=p_{*}\left(z_{i} \frac{\partial}{\partial z_{j}}\right)
$$

for all $0 \leq i<j \leq n$.

Hence the standard Poisson structure on $X=\mathbb{C} \mathbf{P}^{n}$ can be written as

$$
\begin{aligned}
\pi_{s t}=\mu\left(r_{\mathfrak{g}}\right) & =\sum_{0 \leq i<j \leq n} \mu\left(e_{i j}\right) \wedge \mu\left(e_{j i}\right) \\
& =\sum_{0 \leq i<j \leq n} p_{*}\left(z_{j} \frac{\partial}{\partial z_{i}}\right) \wedge p_{*}\left(z_{i} \frac{\partial}{\partial z_{j}}\right) \\
& =\sum_{0 \leq i<j \leq n} p_{*}\left(z_{i} \frac{\partial}{\partial z_{i}}\right) \wedge p_{*}\left(z_{j} \frac{\partial}{\partial z_{j}}\right) \\
& =\sum_{0 \leq i<j \leq n} v_{i} \wedge v_{j} \\
& =\sum_{1 \leq i<j \leq n} v_{i} \wedge v_{j} .
\end{aligned}
$$

The last step holds as $\sum_{i=0}^{n} v_{i}=0$.

\subsection{Exact sequences related to $\mathbb{C P}^{n}$.}

Theorem 2.19. 1] Let $\mathcal{P}$ be a principle bundle over $X$ with group $G$. Then there exists an exact sequence of vector bundles over $X$ :

$$
0 \rightarrow \mathcal{P} \times_{G} \mathfrak{g} \rightarrow T \mathcal{P} / G \rightarrow T X \rightarrow 0,
$$

where $\mathcal{P} \times{ }_{G} \mathfrak{g}$ is the bundle associated to $\mathcal{P}$ by the adjoint representation of $G$ on $\mathfrak{g}=$ Lie $(G)$, and $T \mathcal{P} / G$ is the bundle of invariant vector fields on $\mathcal{P}$. 
Recall that for a principle $G$-bundle $\mathcal{P}$ over $X$, and a representation of $G$ on a vector space $V$, the associated vector bundle over $X$ is defined to be $\mathcal{P} \times{ }_{G} V=(\mathcal{P} \times V) / \sim$, where $(x . g, v) \sim((x, g . v)$, $\forall x \in \mathcal{P}, g \in G, v \in V$.

Let $\mathcal{P}=\mathbb{C}^{n+1} \backslash\{0\}, X=\mathbb{C P}^{n}$, and let $p: \mathcal{P}=\mathbb{C}^{n+1} \backslash\{0\} \rightarrow \mathbb{C P}^{n}$ be the canonical projection. The group $G=\mathbb{C}^{*}$ operates by right multiplication on $\mathcal{P}=\mathbb{C}^{n+1} \backslash\{0\}$ :

$$
\lambda: v \rightarrow v \lambda, \quad v \in \mathbb{C}^{n+1} \backslash\{0\}, \quad \lambda \in \mathbb{C}^{*} .
$$

Then $\mathcal{P}$ is a principle $\mathbb{C}^{*}$-bundle over $X$.

As $G=\mathbb{C}^{*}$ is abelian, the adjoint representation is trivial, we have $\mathcal{P} \times_{G} \mathfrak{g} \cong X \times \mathbb{C}$.

The $\mathbb{C}^{*}$-action on $T \mathcal{P} \cong \mathbb{C}^{n+1} \backslash\{0\} \times \mathbb{C}^{n+1}$ is given by:

$$
(x \times v) \lambda=x \lambda \times v \lambda, \quad x \in \mathbb{C}^{n+1} \backslash\{0\}, v \in \mathbb{C}^{n+1}, \lambda \in \mathbb{C}^{*} .
$$

Hence we have $T \mathcal{P} / G \cong \mathcal{P} \times \mathbb{C}^{*} \mathbb{C}^{n+1} \cong O(1)^{\oplus(n+1)}$, where $\mathcal{P} \times_{\mathbb{C}^{*}} \mathbb{C}^{n+1}$ is the associated bundle of $\mathcal{P}$ by the $\mathbb{C}^{*}$ representation on $\mathbb{C}^{n+1}$ given by:

$$
\rho(\lambda) v=\lambda^{-1} v, \quad v \in \mathbb{C}^{n+1}, \lambda \in \mathbb{C}^{*} .
$$

In this case, the Atiyah exact sequence (2.12) becomes

$$
0 \rightarrow \mathbb{C} \rightarrow O(1)^{\oplus(n+1)} \rightarrow T \mathbb{C} \mathbf{P}^{n} \rightarrow 0,
$$

which is exactly the Euler exact sequence of $\mathbb{C} \mathbf{P}^{n}$.

Suppose that $\left(z_{0}, z_{1}, \ldots, z_{n}\right)$ are the standard coordinates on $\mathbb{C}^{n+1}$. Then the canonical projection $p: \mathbb{C}^{n+1} \backslash\{0\} \rightarrow \mathbb{C P}^{n}$ becomes $\left(z_{0}, z_{1}, \ldots, z_{n}\right) \rightarrow\left[z_{0}, z_{1}, \ldots, z_{n}\right]$. The map $p: \mathcal{P} \rightarrow \mathbb{C P}^{n}$ induces a map $T \mathcal{P} / G \stackrel{p_{*}}{\longrightarrow} T \mathbb{C P}^{n}$. By identification of $T \mathcal{P} / G$ with $O(1)^{\oplus(n+1)}$, and by abuse of notation, we also denote $p_{*}$ by the map $O(1)^{\oplus(n+1)} \rightarrow T \mathbb{C} \mathbf{P}^{n}$ in the Euler sequence (2.13). Let us denote $E$ by the vector bundle $O(1)^{\oplus(n+1)}$, denote $L$ by the kernel of the map $O(1)^{\oplus(n+1)} \stackrel{p_{*}}{\longrightarrow} T \mathbb{C} \mathbf{P}^{n}$. Then $L=\mathbb{C} \vec{e}$ is a subbundle of $E$, where $\vec{e}=\sum_{i=0}^{n} z_{i} \frac{\partial}{\partial z_{i}}$ is the Euler vector field. The Euler exact sequence (2.13) can then be written as

$$
0 \rightarrow L \hookrightarrow E \stackrel{p_{*}}{\longrightarrow} T X \rightarrow 0 .
$$

Let $L \wedge\left(\wedge^{j} E\right)=\mathbb{C} \vec{e} \wedge\left(\wedge^{j} E\right)(0 \leq j \leq n-1)$, that is a subbundle of $\wedge^{j+1} E$. Then we have the following lemma, which is known to Bondal [2].

Lemma 2.20. For $X=\mathbb{C P}^{n}$. we have the exact sequences

$$
0 \rightarrow L \wedge\left(\wedge^{j-1} E\right) \hookrightarrow \wedge^{j} E \stackrel{\vec{e} \wedge \cdot}{\longrightarrow} L \wedge\left(\wedge^{j} E\right) \rightarrow 0
$$

and

$$
0 \rightarrow L \wedge\left(\wedge^{j-1} E\right) \hookrightarrow \wedge^{j} E \stackrel{p_{*}}{\longrightarrow} \wedge^{j} T X \rightarrow 0
$$

for all $j \geq 1$, where

(I) $L \wedge\left(\wedge^{j-1} E\right) \hookrightarrow \wedge^{j} E$ is the embedding of $L \wedge\left(\wedge^{j-1} E\right)$ as a subbundle of $\wedge^{j} E$;

(II) $\wedge^{j} E \stackrel{\vec{e} \wedge \cdot}{\longrightarrow} L \wedge\left(\wedge^{j} E\right)$ is defined by the wedge of $\vec{e}$ with elements in $\wedge^{j} E$;

(II) $\wedge^{j} E \stackrel{p_{*}}{\longrightarrow} \wedge^{j} T X$ is induced by the map $E \stackrel{p_{*}}{\longrightarrow} T X$ in Equation (2.14). 
Proof. At any point $x \in X$, for any $\left.\alpha_{x} \in \wedge^{j} E\right|_{x}$, we have that $\vec{e}_{x} \wedge \alpha_{x}=0$ if and only if there exist $\left.\beta_{x} \in \wedge^{j-1} E\right|_{x}$, such that $\alpha_{x}=\vec{e}_{x} \wedge \beta_{x}$. Thus (2.15) is an exact sequence for all $j \geq 1$.

As we have shown in (2.14), the kernel of $E \stackrel{p_{*}}{\rightarrow} T X$ is the trivial bundle $L=\mathbb{C} \vec{e}$. At any point $x \in X$, the kernel of $\left.\left.E\right|_{x} \stackrel{p_{*}}{\longrightarrow} T X\right|_{x}$ is $\mathbb{C} \vec{e}_{x}$. As a consequence, the kernel of $\left.\left.\wedge^{j} E\right|_{x} \stackrel{p_{*}}{\longrightarrow} \wedge^{j} T X\right|_{x}$ is

$$
\vec{e}_{x} \wedge\left(\left.\wedge^{j-1} E\right|_{x}\right) \text {. }
$$

Thus (2.16) is an exact sequence for all $j \geq 1$.

\section{The Cohomology groups $H^{i}\left(\mathbb{C P}^{n}, \wedge^{j} \mathcal{T}_{\mathbb{C} \mathbf{P}^{n}}\right)$}

3.1. The vanishing of the cohomology group $H^{i}\left(\mathbb{C P}^{n}, \wedge^{j} \mathcal{T}_{\mathbb{C}} \mathbf{P}^{n}\right)$ for $i>0$ and $0 \leq j \leq n$.

Theorem 3.1. For $X=\mathbb{C P}^{n}$, we have

$$
H^{i}\left(X, \wedge^{j} \mathcal{T}_{X}\right)=0
$$

for all $i>0$ and $0 \leq j \leq n$.

Remark 3.2. Theorem 3.1 should be known to Bott [3]. In the case of $j=1$, the conclusion $H^{i}\left(X, \mathcal{T}_{X}\right)=0(i>0)$ is a special case of the Theorem VII in [3].

To make the paper self contained, we will give a proof of Theorem 3.1

Let $L=\mathbb{C} \vec{e}$ and $E=O(1)^{\oplus(n+1)}$ as in Section 2.5. By Lemma 2.20, we get the following lemma.

Lemma 3.3. For $X=\mathbb{C P}^{n}$, we have

$$
H^{i}\left(X, \wedge^{j} T X\right) \cong H^{i}\left(X, L \wedge\left(\wedge^{j} E\right)\right) \cong H^{i+1}\left(X, L \wedge\left(\wedge^{j-1} E\right)\right)
$$

for all $i>0$ and $j \geq 1$.

Proof. The exact sequence (2.15) in Lemma 2.20 induces a long exact sequence

$(3.3) \quad \ldots \rightarrow H^{i}\left(X, \wedge^{j} E\right) \rightarrow H^{i}\left(X, L \wedge\left(\wedge^{j} E\right)\right) \rightarrow H^{i+1}\left(X, L \wedge\left(\wedge^{j-1} E\right)\right) \rightarrow H^{i+1}\left(X, \wedge^{j} E\right) \rightarrow \ldots$

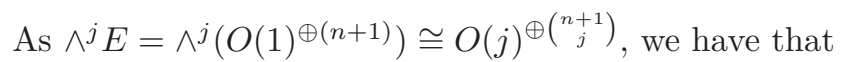

$$
\begin{aligned}
H^{i}\left(X, \wedge^{j} E\right) & =H^{i}\left(X, O(j)^{\oplus\left(\begin{array}{c}
n+1 \\
j
\end{array}\right)}\right) \\
& =H^{i}(X, O(j))^{\oplus\left(\begin{array}{c}
n+1 \\
j
\end{array}\right)} \\
& =H^{i}\left(X, K_{X} \otimes O(n+1+j)\right)^{\oplus\left(\begin{array}{c}
n+1 \\
j
\end{array}\right),}
\end{aligned}
$$

where $K_{X} \cong O(-n-1)$ is the canonical line bundle of $X=\mathbb{C P}^{n}$. By Kodaira vanishing theorem, we have $H^{i}\left(X, K_{X} \otimes O(n+1+j)\right)=0$ for $i>0$, which implies $H^{i}\left(X, \wedge^{j} E\right)=0$ for $i>0$. Thus the exact sequence (3.3) induces the isomorphism

$$
H^{i}\left(X, L \wedge\left(\wedge^{j} E\right)\right) \cong H^{i+1}\left(X, L \wedge\left(\wedge^{j-1} E\right)\right)
$$

for all $i>0$ and $j \geq 1$.

By the similar reason, the exact sequence (2.16) in Lemma 2.20 induces the isomorphism

$$
H^{i}\left(X, \wedge^{j} T X\right) \cong H^{i+1}\left(X, L \wedge\left(\wedge^{j-1} E\right)\right)
$$

for all $i>0$ and $j \geq 1$.

Thus we proved the lemma. 


\section{Proof of Theorem 3.1 ;}

Proof. (1) In the case of $j=0, H^{i}\left(X, \mathcal{O}_{X}\right)=0(i>0)$ is a well known result.

(2) In the case of $j \geq 1$ and $i>0$, by Lemma 3.3, we have that

$$
H^{i}\left(X, \wedge^{j} T X\right) \cong H^{i}\left(X, L \wedge\left(\wedge^{j} E\right)\right) \cong H^{i+1}\left(X, L \wedge\left(\wedge^{j-1} E\right)\right) \cong \ldots \cong H^{i+j}(X, L) .
$$

As $L$ is the trivial line bundle, we have $H^{i+j}(X, L)=H^{i+j}\left(X, O_{X}\right)=0$ for $i>0$ and $j \geq 1$, which implies that

$$
H^{i}\left(X, \wedge^{j} \mathcal{T}_{X}\right)=H^{i+j}(X, L)=0
$$

for all $i>0$ and $j \geq 1$.

3.2. Holomorphic vector fields and multi-vector fields on $\mathbb{C} \mathbf{P}^{n}$. Let us introduce some notations first, which are important for this paper. Our setting are based on Example 2.11 and Example 2.16.

- Let $v_{i}=p_{*}\left(z_{i} \frac{\partial}{\partial z_{i}}\right)=\rho\left(e_{i}\right)(0 \leq i \leq n)$. Denote $W$ as the $n$-dimensional $\mathbb{C}$-vector space generated by $\left\{v_{1}, \ldots, v_{n}\right\}$. Then we have $W=\rho\left(N_{\mathbb{C}}\right)$. Let $W^{k}=\wedge^{k} W(1 \leq k \leq n)$ and $W^{0}=\mathbb{C}$. Then $W^{k}$ can be considered as a subspace of $H^{0}\left(X, \wedge^{k} \mathcal{T}_{X}\right)$.

- Given any $I=\left(m_{1}, \ldots, m_{n}\right) \in M$, let $m_{0}=-\sum_{i=1}^{n} m_{i}$, then $\chi^{m}=z_{0}^{m_{0}} \ldots z_{n}^{m_{n}}$ can be considered as a rational function on $\mathbb{C P}^{n}$. Suppose that $\left\{m_{i_{1}}, m_{i_{2}}, \ldots, m_{i_{l}} \mid 0 \leq i_{1}<i_{2}<\right.$ $\left.\ldots<i_{l} \leq n\right\}$ is the set of all the elements equal to -1 in $\left\{m_{0}, m_{1}, \ldots, m_{n}\right\}$. Set

$$
|I|=l, \quad \chi^{I}=z_{0}^{m_{0}} \ldots z_{n}^{m_{n}}, \quad \mathcal{V}_{I}=v_{i_{1}} \wedge \ldots \wedge v_{i_{l}} \in W^{l}, \quad \mathcal{E}_{I}=e_{i_{1}} \wedge \ldots \wedge e_{i_{l}} \in \wedge^{l} N .
$$

Notice that $v_{0}=-\sum_{i=1}^{n} v_{i}$ and $e_{0}=-\sum_{i=1}^{n} e_{i}$.

The following theorem described the structure of the space of holomorphic vector fields and multivector fields on $\mathbb{C} \mathbf{P}^{n}$ with respect to the $\left(\mathbb{C}^{*}\right)^{n}$-action on $H^{0}\left(X, \wedge^{k} \mathcal{T}_{X}\right)(0 \leq k \leq n)$.

Theorem 3.4. Let $X=\mathbb{C P}^{n}$. Let $S_{k}(0 \leq k \leq n)$ be the set consisting of all $I \in M$ satisfying the conditions

$$
\left\langle I, e_{i}\right\rangle=m_{i} \geq-1 \quad(0 \leq i \leq n)
$$

and

$$
|I| \leq k
$$

Let

$$
V_{I}^{k}=\mathbb{C}\left(\chi^{I} \cdot \mathcal{V}_{I}\right) \wedge W^{k-|I|}
$$

for $|I| \leq k \leq n$. The $\left(\mathbb{C}^{*}\right)^{n}$-action on $X=\mathbb{C P}^{n}$ induced a $\left(\mathbb{C}^{*}\right)^{n}$-representation on $H^{0}\left(X, \wedge^{k} \mathcal{T}_{X}\right)$, which has the weight spaces decomposition

$$
H^{0}\left(X, \wedge^{k} \mathcal{T}_{X}\right)=\bigoplus_{I \in S_{k}} V_{I}^{k}
$$

where $S_{k}$ is the set of all weights, and $V_{I}^{k}$ is the weight space of the weight $I$. 
In Theorem 3.4 $S_{k}$ is the set of all $I \in M$ satisfying conditions 3.4 and 3.5 Let us denote $S(i)$ $(0 \leq i \leq n)$ as the set of all all $I \in M$ satisfying the condition (3.4) and $|I|=i$. Then we have $S_{k}=S_{k-1} \cup S(i)$ and $S_{0} \subseteq S_{1} \subseteq S_{2} \ldots \subseteq S_{n}$. By Theorem 3.4 we have

Proposition 3.5. Let $X=\mathbb{C P}^{n}$. We have

$$
H^{0}\left(X, \wedge{ }^{k} \mathcal{T}_{X}\right)=\left(H^{0}\left(X, \wedge^{k-1} \mathcal{T}_{X}\right) \wedge W\right) \oplus\left(\bigoplus_{I \in S(k)} \mathbb{C} \chi^{I} \cdot \mathcal{V}_{I}\right) \quad(1 \leq k \leq n),
$$

where $S(k)$ is the set of all all $I \in M$ satisfying the condition (3.4) and $|I|=k$.

Next we will prove Theorem 3.4 and Proposition 3.5.

By Lemma 2.20, we have

Lemma 3.6. For $X=\mathbb{C P}^{n}$, we have the exact sequences

$$
0 \rightarrow H^{0}\left(X, L \wedge\left(\wedge^{j-1} E\right)\right) \rightarrow H^{0}\left(X, \wedge^{j} E\right) \stackrel{p_{*}}{\rightarrow} H^{0}\left(X, \wedge^{j} T X\right) \rightarrow 0
$$

for all $j \geq 1$.

Proof. The exact sequence (2.16) induces a long exact sequence

$$
0 \rightarrow H^{0}\left(X, L \wedge\left(\wedge^{j-1} E\right)\right) \rightarrow H^{0}\left(X, \wedge^{j} E\right) \stackrel{p_{*}}{\rightarrow} H^{0}\left(X, \wedge^{j} T X\right) \rightarrow H^{1}\left(X, L \wedge\left(\wedge^{j-1} E\right)\right) \rightarrow \cdots
$$

for all $j \geq 1$. By Lemma 3.3, we have

$$
H^{1}\left(X, L \wedge\left(\wedge^{j-1} E\right)\right) \cong H^{2}\left(X, L \wedge\left(\wedge^{j-2} E\right)\right) \cong \ldots \cong H^{j}(X, L)=H^{j}\left(X, O_{X}\right)=0
$$

for all $j \geq 1$. Therefore we have the exact sequences

$$
0 \rightarrow H^{0}\left(X, L \wedge\left(\wedge^{j-1} E\right)\right) \rightarrow H^{0}\left(X, \wedge^{j} E\right) \stackrel{p_{*}}{\rightarrow} H^{0}\left(X, \wedge^{j} T X\right) \rightarrow 0
$$

for all $j \geq 1$.

Remark 3.7. In the case of $j=1$, the exact sequence (3.9) becomes

$$
0 \rightarrow \mathbb{C} \rightarrow H^{0}(X, O(1))^{\oplus(n+1)} \rightarrow H^{0}\left(X, T_{X}\right) \rightarrow 0 .
$$

As $\wedge^{j} E=O(j)^{\oplus\left(\begin{array}{c}n+1 \\ j\end{array}\right)}$, we have that $H^{0}\left(X, \wedge^{j} E\right) \cong H^{0}(X, O(j))^{\oplus\left(\begin{array}{c}n+1 \\ j\end{array}\right)}$.

Let us denote $F_{j}(1 \leq k \leq n+1)$ by the complex vector space of the $j$-vector fields

$$
\sum_{0 \leq i_{1}<i_{2}<\ldots<i_{k} \leq n} g_{i_{1}, i_{2}, \ldots, i_{j}} \frac{\partial}{\partial z_{i_{1}}} \wedge \ldots \wedge \frac{\partial}{\partial z_{i_{j}}}
$$

on $\mathbb{C}^{n+1}$, where $g_{i_{1}, i_{2}, \ldots, i_{j}}$ are homogenous polynomials with variables $z_{0}, z_{1}, \ldots, z_{n}$ of degree $j$. The restriction of such $j$-vector fields on $\mathcal{P}=\mathbb{C}^{n+1} \backslash\{0\} \subset \mathbb{C}^{n+1}$ form a vector space. We denote it by $\tilde{F}_{j}$. Then the complex vector spaces below are isomorphic:

(a) $F_{j}$;

(b) $\tilde{F}_{j}$;

(c) $H^{0}\left(X, \wedge^{j} E\right)$;

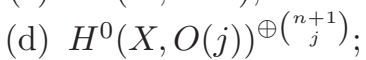

where $1 \leq j \leq n+1$.

By identification of the vector spaces above, and by Lemma 3.6, we have 
Lemma 3.8. 2] Let $p: \mathcal{P}=\mathbb{C}^{n+1} \backslash\{0\} \rightarrow \mathbb{C P}^{n}:\left(z_{0}, z_{1}, \ldots, z_{n}\right) \rightarrow\left[z_{0}, z_{1}, \ldots, z_{n}\right]$ be the canonical projection. Then we have

(1) Any holomorphic k-vector field on $\mathbb{C} \mathbf{P}^{n}$ can be written as

$$
p_{*}\left(\sum_{0 \leq i_{1}<i_{2}<\ldots<i_{k} \leq n} g_{i_{1}, i_{2}, \ldots, i_{k}} \frac{\partial}{\partial z_{i_{1}}} \wedge \ldots \wedge \frac{\partial}{\partial z_{i_{k}}}\right),
$$

where $g_{i_{1}, i_{2}, \ldots, i_{k}}$ are homogenous polynomials with variables $z_{0}, z_{1}, \ldots, z_{n}$ of degree $k$. Or in other words,

$$
H^{0}\left(X, \wedge{ }^{k} \mathcal{T}_{X}\right)=p_{*}\left(\tilde{F}_{k}\right) .
$$

(2) The kernel of the map $p_{*}: \tilde{F}_{k} \rightarrow H^{0}\left(X, \wedge^{k} \mathcal{T}_{X}\right)$ is

$$
\operatorname{ker} p_{*}=\left(\sum_{i=0}^{n} z_{i} \frac{\partial}{\partial z_{i}}\right) \wedge \tilde{F}_{k-1}
$$

\section{Proof of Theorem 3.4;}

Proof. (1) First, we will prove that

$$
H^{0}\left(X, \wedge^{k} \mathcal{T}_{X}\right)=\sum_{I \in S_{k}} V_{I}^{k} \quad(0 \leq k \leq n),
$$

which is equivalent to

$$
H^{0}\left(X, \wedge^{k} \mathcal{T}_{X}\right) \subseteq \sum_{I \in S_{k}} V_{I}^{k}
$$

and

$$
\sum_{I \in S_{k}} V_{I}^{k} \subseteq H^{0}\left(X, \wedge{ }^{k} \mathcal{T}_{X}\right)
$$

(a) By Lemma 3.8 any holomorphic $k$-vector field $\Psi$ on $\mathbb{C} \mathbf{P}^{n}$ can be written as

$$
\begin{aligned}
\Psi & =p_{*}\left(\sum_{0 \leq i_{1}<i_{2}<\ldots<i_{k} \leq n} g_{i_{1}, i_{2}, \ldots, i_{k}} \frac{\partial}{\partial z_{i_{1}}} \wedge \ldots \wedge \frac{\partial}{\partial z_{i_{k}}}\right) \\
& =\sum_{0 \leq i_{1}<i_{2}<\ldots<i_{k} \leq n} f_{i_{1}, i_{2}, \ldots, i_{k}} v_{i_{1}} \wedge \ldots \wedge v_{i_{k}},
\end{aligned}
$$

where $g_{i_{1}, i_{2}, \ldots, i_{k}}$ are homogenous polynomials with variables $z_{0}, z_{1}, \ldots, z_{n}$ of degree $k$, and $f_{i_{1}, i_{2}, \ldots, i_{k}}=\frac{g_{i_{1}, i_{2}, \ldots, i_{k}}}{\prod_{s=1}^{k} z_{i_{s}}}$. Suppose that

$$
f_{i_{1}, i_{2}, \ldots, i_{k}}=\sum c_{m_{0}, \ldots, m_{n}}^{i_{1}, \ldots, i_{k}} z_{0}^{m_{0}} \ldots z_{n}^{m_{n}}
$$

where $c_{m_{0}, \ldots, m_{n}}^{i_{1}, \ldots, i_{k}}$ are complex constants.

The integers $m_{i}(0 \leq i \leq n)$ in Equation (3.12) have to satisfy the conditions below:

(i) $m_{i_{s}} \geq-1$ for $1 \leq s \leq k$,

(ii) $m_{j} \geq 0$ for $j \notin\left\{i_{s} \mid 1 \leq s \leq k\right\}$,

(iii) $\sum_{i=0}^{n} m_{i}=0$. 
It is easy to verify that any element $I=\left(m_{1}, \ldots, m_{n}\right) \in M$ satisfying the conditions (i), (ii) and (iii) satisfies $I \in S_{k}$.

Without loss of generality, suppose that $\left\{m_{i_{1}}, m_{i_{2}}, \ldots, m_{i_{l}} \mid 0 \leq i_{1}<i_{2}<\ldots<i_{l} \leq n\right\}$ is the set of all the elements equal to -1 in $\left\{m_{0}, m_{1}, \ldots, m_{n}\right\}$. Then

$$
\begin{aligned}
z_{0}^{m_{0}} \ldots z_{n}^{m_{n}} v_{i_{1}} \wedge \ldots \wedge v_{i_{k}} & =\chi^{I} \cdot\left(v_{i_{1}} \wedge \ldots \wedge v_{i_{l}}\right) \wedge\left(v_{i_{l+1}} \wedge \ldots \wedge v_{i_{k}}\right) \\
& =\chi^{I} \cdot \mathcal{V}_{I} \wedge\left(v_{i_{l+1}} \wedge \ldots \wedge v_{i_{k}}\right)
\end{aligned}
$$

is a $k$-vector field in the space $V_{I}^{k}=V_{I}^{k}\left(I \in S_{k}\right)$. As

$$
\Psi=\sum c_{m_{0}, \ldots, m_{n}}^{i_{1}, \ldots, i_{k}} z_{0}^{m_{0}} \ldots z_{n}^{m_{n}} v_{i_{1}} \wedge \ldots \wedge v_{i_{k}},
$$

we have that

$$
\Psi \in \sum_{I \in S_{k}} V_{I}^{k} \quad(0 \leq k \leq n)
$$

Thus

$$
H^{0}\left(X, \wedge^{k} \mathcal{T}_{X}\right) \subseteq \sum_{I \in S_{k}} V_{I}^{k} \quad(0 \leq k \leq n)
$$

(b) On the other hand, given an element $I=\left(m_{1}, \ldots, m_{n}\right) \in S_{k} \subset M$, suppose that $\left\{m_{i_{1}}, m_{i_{2}}, \ldots, m_{i_{l}} \mid 0 \leq i_{1}<i_{2}<\ldots<i_{l} \leq n\right\}$ is the set of all the elements equal to -1 in $\left\{m_{0}, m_{1}, \ldots, m_{n}\right\}$. Then we have

$$
\begin{aligned}
\chi^{I} \cdot \mathcal{V}_{I} & =z_{0}^{m_{0}} \ldots z_{n}^{m_{n}} v_{i_{1}} \wedge \ldots \wedge v_{i_{l}} \\
& =z_{0}^{m_{0}} \ldots z_{n}^{m_{n}} p_{*}\left(z_{i_{1}} \frac{\partial}{\partial z_{i_{1}}}\right) \wedge \ldots \wedge p_{*}\left(z_{i_{l}} \frac{\partial}{\partial z_{i_{l}}}\right) \\
& =p_{*}\left(\left(z_{i_{1}} \ldots z_{i_{l}}\right) \cdot\left(z_{0}^{m_{0}} \ldots z_{n}^{m_{n}}\right) \frac{\partial}{\partial z_{i_{1}}} \wedge \ldots \wedge \frac{\partial}{\partial z_{i_{l}}}\right) \\
& =p_{*}\left(\left(\prod_{i \notin\left\{i_{1}, \ldots i_{l}\right\}} z_{i}^{m_{i}}\right) \frac{\partial}{\partial z_{i_{1}}} \wedge \ldots \wedge \frac{\partial}{\partial z_{i_{l}}}\right) .
\end{aligned}
$$

As $m_{i} \geq 0$ for $i \notin\left\{i_{1}, \ldots i_{l}\right\}$, we know that $\prod_{i \notin\left\{i_{1}, \ldots i_{l}\right\}} z_{i}^{m_{i}}$ is a polynominal with variables $z_{0}, z_{1}, \ldots z_{n}$ of degree $l$. By Lemma 3.8, $\chi^{I} \cdot \mathcal{V}_{I}$ is a holomorphic $l$-vector field on $X=\mathbb{C P}^{n}$.

As $W^{k-|I|}=W^{k-l}$ is a subspace of $H^{0}\left(X, \wedge^{k-l} \mathcal{T}_{X}\right)$, we get that $V_{I}^{k}=\mathbb{C}\left(\chi^{I} \cdot \mathcal{V}_{I}\right) \wedge$ $W^{k-|I|}$ is a subspace of $H^{0}\left(X, \wedge{ }^{k} \mathcal{T}_{X}\right)$.

Therefore we have

$$
\sum_{I \in S_{k}} V_{I}^{k} \subseteq H^{0}\left(X, \wedge^{k} \mathcal{T}_{X}\right)
$$

(c) By the arguments above, we proved Equation (3.11).

(2) By Equation (3.11) , it is easy to verify that $V_{I}^{k}=\mathbb{C}\left(\chi^{I} \cdot \mathcal{V}_{I}\right) \wedge W^{k-|I|}$ is the weight space of the weight $I$. Thus we have

$$
H^{0}\left(X, \wedge^{k} \mathcal{T}_{X}\right)=\bigoplus_{I \in S_{k}} V_{I}^{k} \quad(0 \leq k \leq n)
$$

\section{Proof of Proposition 3.5;}


Proof. Since $S_{k}=S_{k-1} \cup S(k)$, by Theorem 3.4, we have

$$
\begin{gathered}
H^{0}\left(X, \wedge{ }^{k} \mathcal{T}_{X}\right)=\bigoplus_{I \in S_{k}} \mathbb{C}\left(\chi^{I} \cdot \mathcal{V}_{I}\right) \wedge W^{k-|I|} \\
=\left(\bigoplus_{I \in S_{k-1}} \mathbb{C}\left(\chi^{I} \cdot \mathcal{V}_{I}\right) \wedge W^{k-|I|}\right) \oplus\left(\bigoplus_{I \in S(k)} \mathbb{C} \chi^{I} \cdot W^{k}\right) .
\end{gathered}
$$

As $W^{k-|I|}=W^{k-1-|I|} \wedge W$, by Theorem 3.4, we have

$$
\begin{gathered}
\bigoplus_{I \in S_{k-1}} \mathbb{C}\left(\chi^{I} \cdot \mathcal{V}_{I}\right) \wedge W^{k-|I|}=\left(\bigoplus_{I \in S_{k-1}} \mathbb{C}\left(\chi^{I} \cdot \mathcal{V}_{I}\right) \wedge W^{k-1-|I|}\right) \wedge W \\
=H^{0}\left(X, \wedge^{k-1} \mathcal{T}_{X}\right) \wedge W .
\end{gathered}
$$

Therefore we have

$$
H^{0}\left(X, \wedge^{k} \mathcal{T}_{X}\right)=\left(H^{0}\left(X, \wedge^{k-1} \mathcal{T}_{X}\right) \wedge W\right) \oplus\left(\bigoplus_{I \in S(k)} \mathbb{C} \chi^{I} \cdot W^{k}\right) \quad(1 \leq k \leq n)
$$

\section{Poisson cohomology of $\mathbb{C P}^{n}$}

4.1. Poisson cohomology of toric Poisson structures on $\mathbb{C} \mathbf{P}^{n}$. Let us introduce some necessary notations first.

- Let $\pi=\sum_{1 \leq i<j \leq n} a_{i j} v_{i} \wedge v_{j}$ be a holomorphic toric Poisson structure on $X=\mathbb{C P}^{n}$, where $v_{i}=p_{*}\left(z_{i} \frac{\partial}{\partial z_{i}}\right)=\rho\left(e_{i}\right)(0 \leq i \leq n)$. We define $\Pi \in \wedge^{2} N_{\mathbb{C}}$ by the equation

$$
\rho(\Pi)=\pi \text {. }
$$

Then

$$
\Pi=\sum_{1 \leq i<j \leq n} a_{i j} e_{i} \wedge e_{j} .
$$

- Given $I=\left(m_{1}, \ldots, m_{n}\right) \in M$, we have

$$
\imath_{I} \Pi \in N_{\mathbb{C}}=N \otimes \mathbb{C},
$$

where $\imath_{I} \Pi$ denotes the contraction of $I \in M$ with $\Pi \in \wedge^{2} N_{\mathbb{C}}$. Let $m_{0}=-\sum_{i=1}^{n} m_{i}$. Then $\chi^{I}=z_{0}^{m_{0}} z_{1}^{m_{1}} \cdots z_{n}^{m_{n}}$ can be considered as a rational function on $X=\mathbb{C P}^{n}$.

By Lemma 2.4 and Theorem [3.1, the Poisson cohomology of $\left(X=\mathbb{C P}^{n}, \pi\right)$ is isomorphic to the cohomology of the complex

$$
H^{0}\left(X, \mathcal{O}_{X}\right) \stackrel{d_{\pi}}{\longrightarrow} H^{0}\left(X, T_{X}\right) \stackrel{d_{\pi}}{\longrightarrow} H^{0}\left(X, \wedge^{2} T_{X}\right) \stackrel{d_{\pi}}{\longrightarrow} \ldots \stackrel{d_{\pi}}{\longrightarrow} H^{0}\left(X, \wedge^{n} T_{X}\right)
$$

where $d_{\pi}=[\pi, \cdot]$. By Lemma 2.17, there is a $\left(\mathbb{C}^{*}\right)^{n}$-action on $H_{\pi}^{\bullet}(X)$. The following theorem described the Poisson cohomology group $H_{\pi}^{\bullet}(X)$ by the decomposition of the weight spaces of the $\left(\mathbb{C}^{*}\right)^{n}$-respresentation on $H_{\pi}^{\bullet}(X)$.

Theorem 4.1. Let $\pi$ be a holomorphic toric Poisson structure on $X=\mathbb{C} \mathbf{P}^{n}$. Let $V_{I}^{k}=\mathbb{C}\left(\chi^{I}\right.$. $\left.\mathcal{V}_{I}\right) \wedge W^{k-|I|}$ for $|I| \leq k \leq n$. Then we have 
(1) for $0 \leq k \leq n$,

$$
H_{\pi}^{k}(X)=\bigoplus_{I \in S_{k}(\pi)} V_{I}^{k}
$$

where $S_{k}(\pi)$ is the set consisting of all $I \in M$ satisfying

$$
\begin{gathered}
\left\langle I, e_{i}\right\rangle=m_{i} \geq-1 \quad(0 \leq i \leq n) ; \\
|I| \leq k ;
\end{gathered}
$$

and the equation

(2) $H_{\pi}^{k}(X)=0$ for $k>n$.

Remark 4.2. (1) $S_{k}(\pi)$ is a subset of $S_{k}$ consisting of all $I \in S_{k}$ satisfying Equation (4.6). By

Theorem 3.4, $\bigoplus_{I \in S_{k}(\pi)} V_{I}^{k}$ is a subspace of $H^{0}\left(X, \wedge{ }^{k} T_{X}\right)$. In Theorem 4.1, the elements in $\bigoplus_{I \in S_{k}(\pi)} V_{I}^{k}$ represent the cohomology classes in the quotient space

$$
\frac{\text { ker }: H^{0}\left(X, \wedge^{k} T_{X}\right) \stackrel{d_{\pi}}{\longrightarrow} H^{0}\left(X, \wedge^{k+1} T_{X}\right)}{\operatorname{Im}: H^{0}\left(X, \wedge^{k-1} T_{X}\right) \stackrel{d_{\pi}}{\longrightarrow} H^{0}\left(X, \wedge^{k} T_{X}\right)} .
$$

(2) For $k=0, H_{\pi}^{0}(X)=\mathbb{C}$ consist of complex constants.

(3) For $k=n$, Theorem 4.1 can be state in the following equivalent way:

$$
H_{\pi}^{n}(X)=\oplus_{I} \mathbb{C} \chi^{I} \cdot v_{1} \wedge \ldots \wedge v_{n}
$$

for all $I \in S_{n} \subset M$ satisfying one of the following conditions

$$
\left\{\begin{array}{l}
|I|=n, \\
\left(\imath_{I} \Pi\right) \wedge \mathcal{E}_{I}=0 .
\end{array}\right.
$$

Let us denoted $S(i, \pi)$ as the set of all $I \in M$ satisfying $|I|=i$ and the conditions (4.4) and (4.6). By the similar method as we have done in Proposition 3.5 we can prove the following proposition.

Proposition 4.3. Let $\pi$ be a holomorphic toric Poisson structure on $X=\mathbb{C P}^{n}$. For $1 \leq k \leq n$, we have

$$
H_{\pi}^{k}(X)=\left(H_{\pi}^{k-1}(X) \wedge W\right) \oplus\left(\bigoplus_{I \in S(k, \pi)} \mathbb{C}\left(\chi^{I} \cdot \mathcal{V}_{I}\right)\right)
$$

where $S(k, \pi)$ is the set of all $I \in M$ satisfying $|I|=k$ and the conditions (4.4) and (4.6).

We need some lemmas to prove Theorem 4.1

Lemma 4.4. Let $\pi=\sum_{1 \leq i<j \leq n} a_{i j} v_{i} \wedge v_{j}$ be a holomorphic toric Poisson structure on $X=\mathbb{C P}^{n}$. For any $I \in M$, we have that

$$
\begin{gathered}
{\left[\pi, \chi^{I}\right]=\chi^{I} \cdot \rho\left(\imath_{I} \Pi\right)} \\
{\left[\pi, \chi^{I} \cdot \mathcal{V}_{I}\right]=\rho\left(\imath_{I} \Pi\right) \wedge\left(\chi^{I} \cdot \mathcal{V}_{I}\right)=\chi^{I} \cdot \rho\left(\left(\imath_{I} \Pi\right) \wedge \mathcal{E}_{I}\right) ;} \\
{\left[\pi, \chi^{I} \cdot \mathcal{V}_{I} \wedge w\right]=\rho\left(\imath_{I} \Pi\right) \wedge\left(\chi^{I} \cdot \mathcal{V}_{I} \wedge w\right) ;}
\end{gathered}
$$

where $w \in W^{k-|I|}$ and $|I| \leq k \leq n$. 
Proof. (I) For any $I \in M$, we have

$$
\left[v_{i}, \chi^{I}\right]=v_{i}\left(\chi^{I}\right)=\left\langle e_{i}, I\right\rangle \chi^{I}
$$

for $0 \leq i \leq n$. By Equation (4.13), we can prove Equation (4.10) with a simple computation.

(II) As $T_{N} \cong\left(\mathbb{C}^{*}\right)^{n}$ is commutative, we have

for $0 \leq i, j \leq n$.

By Equation (4.10) and Equation (4.14), we have

$$
\left[\pi, \chi^{I} \cdot \mathcal{V}_{I}\right]=\rho\left(\imath_{I} \Pi\right) \wedge\left(\chi^{I} \cdot \mathcal{V}_{I}\right)
$$

As $\rho\left(\mathcal{E}_{I}\right)=\mathcal{V}_{I}$, we have

$$
\left[\pi, \chi^{I} \cdot \mathcal{V}_{I}\right]=\rho\left(\imath_{I} \Pi\right) \wedge\left(\chi^{I} \cdot \mathcal{V}_{I}\right)=\chi^{I} \cdot \rho\left(\left(\imath_{I} \Pi\right) \wedge \mathcal{E}_{I}\right) .
$$

(III) As a consequence of Equation (4.11) and Equation (4.14), we can get Equation (4.12).

By Theorem 3.4 and Lemma 4.4, we have given a description of the operator $d_{\pi}$ in the complex

$$
H^{0}\left(X, \mathcal{O}_{X}\right) \stackrel{d_{\pi}}{\longrightarrow} H^{0}\left(X, T_{X}\right) \stackrel{d_{\pi}}{\longrightarrow} H^{0}\left(X, \wedge^{2} T_{X}\right) \stackrel{d_{\pi}}{\longrightarrow} \ldots \stackrel{d_{\pi}}{\longrightarrow} H^{0}\left(X, \wedge^{n} T_{X}\right) .
$$

Lemma 4.5. Let $\pi$ be a holomorphic toric Poisson structure on $X=\mathbb{C} \mathbf{P}^{n}$. We have

$$
d_{\pi}\left(V_{I}^{k}\right) \subseteq V_{I}^{k+1}
$$

for all $I \in S_{k}(0 \leq k \leq n)$, where

(1) $V_{I}^{k}$ is considered as a subspace of $H^{0}\left(X, \wedge{ }^{k} T_{X}\right)$;

(2) $d_{\pi}\left(V_{I}^{k}\right)$ denotes the image of $V_{I}^{k}$ under the map $H^{0}\left(X, \wedge^{k} T_{X}\right) \stackrel{d_{\pi}}{\longrightarrow} H^{0}\left(X, \wedge^{k+1} T_{X}\right)$;

(3) $V_{I}^{k+1}$ is considered as a subspace of $H^{0}\left(X, \wedge^{k+1} T_{X}\right)$.

Proof. Let $\Psi=\chi^{I} \cdot \mathcal{V}_{I} \wedge w$ be a holomorphic $k$-vector field in $V_{I}^{k}$, where $I \in S_{k}$ and $w \in W^{k-|I|}$. By Lemma 4.4, we have

$$
\begin{gathered}
d_{\pi}(\Psi)=\left[\pi, \chi^{I} \cdot \mathcal{V}_{I} \wedge w\right]=\chi^{I} \cdot \rho\left(\imath_{I} \Pi\right) \wedge\left(\mathcal{V}_{I} \wedge w\right) \\
=(-1)^{|I|} \chi^{I} \cdot \mathcal{V}_{I} \wedge\left(\rho\left(\iota_{I} \Pi\right) \wedge w\right) .
\end{gathered}
$$

As $\rho\left(\iota_{I} \Pi\right) \wedge w \in W^{k-|I|+1}, d_{\pi}(\Psi)$ is an element in $V_{I}^{k+1}$.

Thus

$$
d_{\pi}\left(V_{I}^{k}\right) \subseteq V_{I}^{k+1} \quad \text { for all } \quad I \in S_{k}
$$

By Lemma 4.5, we have the chain complex

$$
\mathbb{C}\left(\chi^{I} \cdot \mathcal{V}_{I}\right) \stackrel{d_{\pi}}{\longrightarrow} \mathbb{C}\left(\chi^{I} \cdot \mathcal{V}_{I}\right) \wedge W \stackrel{d_{\pi}}{\longrightarrow} \mathbb{C}\left(\chi^{I} \cdot \mathcal{V}_{I}\right) \wedge W^{2} \stackrel{d_{\pi}}{\longrightarrow} \cdots \stackrel{d_{\pi}}{\longrightarrow} \mathbb{C}\left(\chi^{I} \cdot \mathcal{V}_{I}\right) \wedge W^{n-|I|}
$$

for all $I \in M$, where $\mathbb{C}\left(\chi^{I} \cdot \mathcal{V}_{I}\right) \wedge W^{k}$ is considered as a subspace of $H^{0}\left(X, \wedge^{|I|+k} T X\right)$ for $0 \leq k \leq$ $n-|I|$.

Lemma 4.6. Let $\pi$ be a holomorphic toric Poisson structure on $X=\mathbb{C P}^{n}$. 
(1) Given any $I \in S_{k}(\pi)(0 \leq k \leq n)$, ı.e., $I \in S_{k}$ satisfying the equation

$$
\left(\imath_{I} \Pi\right) \wedge \mathcal{E}_{I}=0,
$$

we have

$$
d_{\pi}\left(V_{I}^{k}\right)=0
$$

(2) Given any $I \in S_{k-1} \subset S_{k}(1 \leq k \leq n)$ satisfying the equation

$$
\left(\iota_{I} \Pi\right) \wedge \mathcal{E}_{I} \neq 0 \text {, }
$$

if $\Psi$ is a holomorphic $k$-vector field in $V_{I}^{k}$ satisfying

$$
d_{\pi}(\Psi)=0,
$$

then there exists a holomorphic $(k-1)$-vector field $\Phi$ in $V_{I}^{k-1}$, such that

$$
\Psi=d_{\pi}(\Phi) .
$$

Proof. (1) For any $I \in S_{k}$ satisfying the equation

$$
\left(\imath_{I} \Pi\right) \wedge \mathcal{E}_{I}=0,
$$

we have

$$
\rho\left(\left(\imath_{I} \Pi\right) \wedge \mathcal{E}_{I}\right)=\rho\left(\imath_{I} \Pi\right) \wedge \mathcal{V}_{I}=0 .
$$

By Equation (4.12) in Lemma 4.4, we have

$$
d_{\pi}\left(V_{I}^{k}\right)=0
$$

(2) Suppose that $\Psi=\chi^{I} \cdot \mathcal{V}_{I} \wedge w$, where $I \in S_{k-1} \subseteq S_{k}$ and $w \in W^{k-|I|}$.

By Lemma 4.4, we have

$$
\begin{aligned}
d_{\pi}(\Psi) & =\left[\pi, \chi^{I} \cdot \mathcal{V}_{I} \wedge w\right]=\rho\left(\imath_{I} \Pi\right) \wedge\left(\chi^{I} \cdot \mathcal{V}_{I} \wedge w\right) \\
& =\chi^{I} \cdot \rho\left(\imath_{I} \Pi\right) \wedge v_{i_{1}} \wedge \ldots \wedge v_{i_{l}} \wedge w .
\end{aligned}
$$

The condition $d_{\pi}(\Psi)=0$ implies

$$
\rho\left(\imath_{I} \Pi\right) \wedge v_{i_{1}} \wedge \ldots \wedge v_{i_{l}} \wedge w=0 .
$$

And the condition $\left(\imath_{I} \Pi\right) \wedge \mathcal{E}_{I} \neq 0$ implies that

- ${ }_{I} \Pi$ and $e_{i_{1}}, e_{i_{2}} \ldots e_{i_{l}}$ are $\mathbb{C}$-linear independent vectors in $N_{\mathbb{C}}$;

- $\rho\left(\imath_{I} \Pi\right)$ and $v_{i_{1}}, v_{i_{2}} \ldots v_{i_{l}}$ are $\mathbb{C}$-linear independent vectors in $W=\rho\left(N_{\mathbb{C}}\right)$.

By simple linear algebra we know that $w \in W^{k-|I|}$ can be written as

$$
w=\rho\left(i_{I} \Pi\right) \wedge w_{0}+\sum_{s=1}^{l} v_{i_{s}} \wedge w_{i},
$$

where $w_{0}, w_{1}, \ldots, w_{l}$ are elements in $W^{k-|I|-1}$. As a consequence, we have

$$
\begin{aligned}
\Psi & =\chi^{I} \cdot \mathcal{V}_{I} \wedge w \\
& =\chi^{I}\left(v_{i_{1}} \wedge v_{i_{2}} \wedge \ldots \wedge v_{i_{l}}\right) \wedge\left(\rho\left(\imath_{I} \Pi\right) \wedge w_{0}+\sum_{s=1}^{l} v_{i_{s}} \wedge w_{s}\right) \\
& =\chi^{I}\left(v_{i_{1}} \wedge v_{i_{2}} \wedge \ldots \wedge v_{i_{l}}\right) \wedge\left(\rho\left(\imath_{I} \Pi\right) \wedge w_{0}\right) \\
& =(-1)^{|I|} \rho\left(\imath_{I} \Pi\right) \wedge\left(\chi^{I} \cdot \mathcal{V}_{I} \wedge w_{0}\right)
\end{aligned}
$$


Let $\Phi=(-1)^{|I|} \chi^{I} \cdot \mathcal{V}_{I} \wedge w_{0}$. Then $\Phi$ is a holomorphic $(k-1)$-vector field in the space $V_{I}^{k-1}$. And by Lemma 4.4, we have

$$
\Psi=d_{\pi}(\Phi) .
$$

\section{Proof of Theorem 4.1;}

Proof. (1) In the case of $k=0, S_{0}$ consists of only $(0, \ldots 0) \in M$, and $W^{k-|I|}=W^{0}=\mathbb{C}$. Hence we have

$$
H_{\pi}^{0}(X)=\mathbb{C}
$$

(2) In the case of $1 \leq k \leq n$, by Theorem 3.4 any holomorphic $k$-vector field $\Psi \in H^{0}\left(X, \wedge{ }^{k} T_{X}\right)$ can be written as

$$
\Psi=\sum_{I \in S_{k}} \Psi_{I}, \quad \Psi_{I} \in V_{I}^{k}
$$

As

$$
d_{\pi}(\Psi)=\sum_{I \in S_{k}} d_{\pi}\left(\Psi_{I}\right)
$$

by Lemma 4.5 and Theorem 3.4, we have that

$$
d_{\pi}(\Psi)=0 \Longleftrightarrow d_{\pi}\left(\Psi_{I}\right)=0 \quad \text { for all } \quad I \in S_{k} .
$$

(a) For any $I \in S_{k-1} \subseteq S_{k}$ satisfying $\left(\imath_{I} \Pi\right) \wedge \mathcal{E}_{I} \neq 0$, by Lemma 4.6, $d_{\pi}\left(\Psi_{I}\right)=0$ imples that there exist $\Phi_{I} \in V_{I}^{k-1}$ such that $\Psi_{I}=d_{\pi}\left(\Phi_{I}\right)$. Thus there exists only zero Poisson cohomology class in $V_{I}^{k}$ for $I \in S_{k-1} \subseteq S_{k}$ satisfying $\left(\imath_{I} \Pi\right) \wedge \mathcal{E}_{I} \neq 0$.

(b) For any $I \in S_{k-1} \subseteq S_{k}$ satisfying $\left(\imath_{I} \Pi\right) \wedge \mathcal{E}_{I}=0$, i.e., $I \in S_{k-1}(\pi)$, by Lemma 4.6, we have that

$$
d_{\pi}\left(V_{I}^{k-1}\right)=0 \quad \text { and } \quad d_{\pi}\left(V_{I}^{k}\right)=0 .
$$

By Lemma 4.6 and Theorem 3.4, any nonzero element $\Psi_{I} \in V_{I}^{k}$ satisfying $I \in S_{k-1}(\pi)$ represents a nonzero cohomology class in the Poisson cohomology group. Moreover, the space

$$
\bigoplus_{I \in S_{k-1}(\pi)} V_{I}^{k}
$$

can be considered as a subgroup of the Poisson cohomology group $H_{\pi}^{k}(X)$.

(c) For any $I \in S_{k}(\pi) \backslash S_{k-1}(\pi)$, i.e, $I \in S(k)$ satisfying $\left(\imath_{I} \Pi\right) \wedge \mathcal{E}_{I}=0$, by Lemma 4.6, we have

$$
d_{\pi}\left(V_{I}^{k}\right)=0
$$

By Theorem 3.4, we have

$$
H^{0}\left(X, \wedge^{k-1} T_{X}\right)=\bigoplus_{I \in S_{k-1}} V_{I}^{k-1} .
$$

And by Lemma 4.5, we have

$$
d_{\pi}\left(H^{0}\left(X, \wedge^{k-1} T_{X}\right)\right) \subseteq \bigoplus_{I \in S_{k-1}} V_{I}^{k}
$$


Thus any nonzero element $\Psi_{I} \in V_{I}^{k}$ satisfying $I \in S_{k}(\pi) \backslash S_{k-1}(\pi)$ represents a nonzero Poisson cohomology class. Moreover, the space

$$
\bigoplus_{I \in S_{k}(\pi) \backslash S_{k-1}(\pi)} V_{I}^{k}
$$

can be considered as a subgroup of the Poisson cohomology group $H_{\pi}^{k}(X)$. By the arguments above, we have

$$
H_{\pi}^{k}(X)=\bigoplus_{I \in S_{k}(\pi)} V_{I}^{k}
$$

for $1 \leq k \leq n$.

(3) In the case of $k>n, H_{\pi}^{k}(X)=0$ comes directly from Lemma 2.4 and Theorem 3.1

4.2. Poisson cohomology of the standard Poisson structure on $\mathbb{C} \mathbf{P}^{n}$. By Proposition 2.18 the standard Poisson structure on $X=\mathbb{C P}^{n}$ can be written as

$$
\pi_{s t}=\sum_{1 \leq i<j \leq n} v_{i} \wedge v_{j}
$$

where $v_{i}=p_{*}\left(z_{i} \frac{\partial}{\partial z_{i}}\right)=\rho\left(e_{i}\right)(0 \leq i \leq n)$. And we have $\Pi_{s t}=\sum_{1 \leq i<j \leq n} e_{i} \wedge e_{j} \in \wedge^{2} N_{\mathbb{C}}$.

We can apply Theorem 4.1 to compute the Poisson cohomology of the standard Poisson structure on $\mathbb{C} \mathbf{P}^{n}$. Here we only list the Poisson cohomology groups in the case $n=2$ and $n=3$. For other cases it could be done similarly, but the computation will be more complicated.

Proposition 4.7. The standard Poisson structure on $X=\mathbb{C P}^{2}$ can be written as

$$
\pi_{s t}=v_{1} \wedge v_{2}
$$

The Poisson cohomology groups of $\left(X, \pi_{s t}\right)$ are as following:

(1) $H_{\pi_{s t}}^{0}(X)=\mathbb{C}, \operatorname{dim} H_{\pi_{s t}}^{0}(X)=1$.

(2) $H_{\pi_{s t}}^{1}(X)$ has a basis $\left\{v_{1}, v_{2}\right\}$, $\operatorname{dim} H_{\pi_{s t}}^{1}(X)=2$.

(3) $H_{\pi_{s t}}^{2}(X)$ has a basis $\left\{\left(z_{0}^{m_{0}} z_{1}^{m_{1}} z_{2}^{m_{2}}\right) v_{1} \wedge v_{2}\right\}$ with $\left(m_{0}, m_{1}, m_{2}\right)$ in the set

$$
\left\{\begin{array}{c}
(0,0,0), \\
(-1,-1,2), \quad(-1,2,-1), \quad(2,-1,-1)
\end{array}\right\} .
$$

Hence $\operatorname{dim} H_{\pi_{s t}}^{2}(X)=4$.

(4) $H_{\pi_{s t}}^{k}(X)=0$ for $k>2$.

The Proposition 4.7 verified the results about Poisson cohomology of $\mathbb{C} \mathbf{P}^{2}$ in [15].

Proposition 4.8. The standard Poisson structure on $X=\mathbb{C} \mathbf{P}^{3}$ can be written as

$$
\pi_{s t}=v_{1} \wedge v_{2}+v_{1} \wedge v_{3}+v_{2} \wedge v_{3} .
$$

The Poisson cohomology groups of $\left(X, \pi_{s t}\right)$ are as following:

(1) $H_{\pi_{s t}}^{0}(X)=\mathbb{C}, \operatorname{dim} H_{\pi_{s t}}^{0}(X)=1$.

(2) $H_{\pi_{s t}}^{1}(X)$ has a basis $\left\{v_{1}, v_{2}, v_{3}\right\}$, $\operatorname{dim} H_{\pi_{s t}}^{1}(X)=3$.

(3) $H_{\pi_{s t}}^{2}(X)$ has a basis, which is the union of the three parts:

(a) $\left\{v_{1} \wedge v_{2}, \quad v_{1} \wedge v_{3}, \quad v_{2} \wedge v_{3}\right\}$ 
(b) $\left\{\left(z_{0}^{m_{0}} z_{1}^{m_{1}} z_{2}^{m_{2}} z_{3}^{m_{3}}\right) v_{0} \wedge v_{2}\right\}$ with $\left(m_{0}, m_{1}, m_{2}, m_{3}\right)$ in the set

$$
\{(-1,1,,-1,1),(-1,2,-1,0),(-1,0,-1,2)\}
$$

and $v_{0}=-\sum_{i=1}^{3} v_{i}$

(c) $\left\{\left(z_{0}^{m_{0}} z_{1}^{m_{1}} z_{2}^{m_{2}} z_{3}^{m_{3}}\right) v_{1} \wedge v_{3}\right\}$ with $\left(m_{0}, m_{1}, m_{2}, m_{3}\right)$ in the set

$$
\{(1,-1,1,-1),(2,-1,0,-1),(0,-1,2,-1)\} .
$$

Hence $\operatorname{dim} H_{\pi_{s t}}^{2}(X)=9$.

(4) $H_{\pi_{s t}}^{3}(X)$ has a basis $\left\{\left(z_{0}^{m_{0}} z_{1}^{m_{1}} z_{2}^{m_{2}} z_{3}^{m_{3}}\right) v_{1} \wedge v_{2} \wedge v_{3}\right\}$ with $\left(m_{0}, m_{1}, m_{2}, m_{3}\right)$ in the set

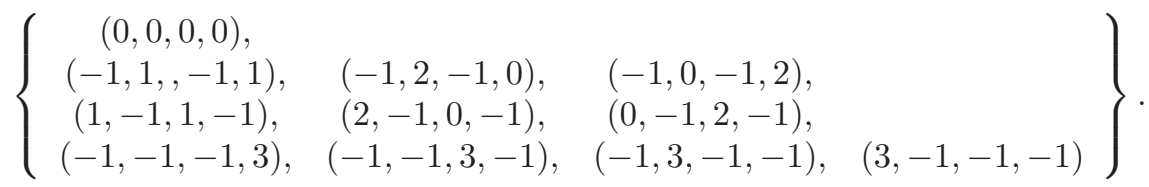

Hence $\operatorname{dim} H_{\pi_{s t}}^{3}(X)=11$.

(5) $H_{\pi_{s t}}^{k}(X)=0$ for $k>3$.

For general $\mathbb{C P}^{n}$ equipped with the standard Poisson structure, we can compute the Poisson cohomology groups by Theorem 4.1. Here we have a result for the first Poisson cohomology group of $\left(\mathbb{C P}^{n}, \pi_{s t}\right)$.

Theorem 4.9. For $X=\mathbb{C P}^{n}$ equipped with the standard Poisson structure

$$
\pi_{s t}=\sum_{1 \leq i<j \leq n} v_{i} \wedge v_{j}
$$

we have

$$
H_{\pi_{s t}}^{1}(X)=W \quad \text { and } \quad \operatorname{dim} H_{\pi_{s t}}^{1}(X)=n .
$$

To prove Theorem 4.9, we need the following lemma.

Lemma 4.10. For $X=\mathbb{C P}^{n}$ equipped with the standard Poisson structure

$$
\pi_{s t}=\sum_{1 \leq i<j \leq n} v_{i} \wedge v_{j}
$$

we have

$$
S\left(1, \pi_{s t}\right)=\emptyset,
$$

where $S\left(1, \pi_{s t}\right)$ is the set of all $I \in S(1)$ satisfying the Equation

$$
\left(\imath_{I} \Pi_{s t}\right) \wedge \mathcal{E}_{I}=0
$$

here $\Pi_{s t}=\sum_{1 \leq i<j \leq n} e_{i} \wedge e_{j}$.

Proof. Let us denote $\alpha_{i, j}(0 \leq i \neq j \leq n)$ as the element in $M$, with $m_{i}=-1, m_{j}=1$ and 0 elsewhere. Then we have

$$
S(1)=\left\{\alpha_{i, j} \mid 0 \leq i \neq j \leq n\right\}
$$

In the case of $I=\alpha_{i, j}$, we have $\mathcal{E}_{I}=e_{i}$. The Equation (4.18) becomes

$$
\left(\imath_{I} \Pi_{s t}\right) \wedge e_{i}=0
$$

which is equivalent to

$$
\imath_{I} \Pi_{s t}=\lambda e_{i}, \quad \lambda \in \mathbb{C} .
$$


Suppose that $\left\{e_{1}^{*}, e_{2}^{*}, \ldots, e_{n}^{*}\right\} \subset M$ is the dual basis of $\left\{e_{1}, e_{2}, \ldots, e_{n}\right\}$. Then we have

$$
I= \begin{cases}e_{j}^{*}, & \text { for } i=0, j \neq 0 . \\ -e_{i}^{*}, & \text { for } i \neq 0, j=0 . \\ -e_{i}^{*}+e_{j}^{*}, & \text { for } i \neq 0, j \neq 0 .\end{cases}
$$

(a) In the case $i=0$ and $j \neq 0$, Equation (4.20) becomes

$$
\imath_{e_{j}^{*}} \Pi=\lambda e_{0}=-\lambda \sum_{k=1}^{n} e_{k},
$$

which implies

$$
\Pi\left(e_{j}^{*}, e_{k}^{*}\right)=\left\langle\imath_{e_{j}^{*}} \Pi, e_{k}^{*}\right\rangle=\left\langle-\lambda \sum_{k=1}^{n} e_{k}, e_{k}^{*}\right\rangle=-\lambda
$$

for all $1 \leq k \leq n$.

But

$$
\Pi_{s t}=\sum_{1 \leq s<t \leq n} e_{s} \wedge e_{t}
$$

which imples

$$
\Pi\left(e_{j}^{*}, e_{j}^{*}\right)=0
$$

and

$$
\Pi\left(e_{j}^{*}, e_{k}^{*}\right)= \pm 1
$$

for all $1 \leq k \neq j \leq n$. Thus Equation (4.21) can not hold for all $1 \leq k \leq n$.

So Equation (4.20) has no solution in this case.

(b) In the case $i \neq 0$ and $j=0$, Equation (4.20) becomes

which implies

$$
\imath_{\left(-e_{i}^{*}\right)} \Pi=\lambda e_{i},
$$

for all $1 \leq k \neq i \leq n$.

$$
\Pi\left(-e_{i}^{*}, e_{k}^{*}\right)=0
$$

But

$$
\Pi_{s t}=\sum_{1 \leq s<t \leq n} e_{s} \wedge e_{t}
$$

which implies

$$
\Pi\left(-e_{i}^{*}, e_{k}^{*}\right)= \pm 1
$$

for all $1 \leq k \neq i \leq n$.

Thus Equation (4.20) has no solution in this case.

(c) In the case $i \neq 0$ and $j \neq 0, I=-e_{i}^{*}+e_{j}^{*}$, Equation (4.20) becomes

$$
\imath_{\left(-e_{i}^{*}+e_{j}^{*}\right)} \Pi=\lambda e_{i} .
$$

It can not be true since that

$$
\Pi\left(-e_{i}^{*}+e_{j}^{*}, e_{j}^{*}\right)=\Pi\left(-e_{i}^{*}, e_{j}^{*}\right)= \pm 1,
$$

but

$$
\left\langle\lambda e_{i}, e_{j}^{*}\right\rangle=0 .
$$

By the arguments above, we have $S\left(1, \pi_{s t}\right)=\emptyset$. 


\section{Proof of Theorem 4.9;}

Proof. By Theorem 4.1, we have

$$
H_{\pi_{s t}}^{1}(X)=\bigoplus_{I \in S_{1}\left(\pi_{s t}\right)} \mathbb{C}\left(\chi^{I} \cdot \mathcal{V}_{I}\right) \wedge W^{1-|I|}
$$

By Lemma 4.10, $S\left(1, \pi_{s t}\right)=\emptyset$, we have $S_{1}\left(\pi_{s t}\right)=S\left(0, \pi_{s t}\right) \cup S\left(1, \pi_{s t}\right)=S\left(0, \pi_{s t}\right)$.

As $S\left(0, \pi_{s t}\right)$ consists only one element $I=(0, \ldots, 0)$, we have

$$
\bigoplus_{I \in S\left(0, \pi_{s t}\right)} \mathbb{C}\left(\chi^{I} \cdot \mathcal{V}_{I}\right) \wedge W^{1-|I|}=W
$$

Therefore we have

$$
H_{\pi_{s t}}^{1}(X)=W \quad \text { and } \quad \operatorname{dim} H_{\pi_{s t}}^{1}(X)=n
$$

Let $\sigma: \mathbb{C} \mathbf{P}^{n} \rightarrow \mathbb{C} \mathbf{P}^{n}$ be an isomorphism of $\mathbb{C} \mathbf{P}^{n}$ defined by

$$
\sigma\left(\left[z_{0}, z_{1}, \ldots, z_{n-1}, z_{n}\right]\right)=\left[z_{1}, z_{2}, \ldots, z_{n}, z_{0}\right] .
$$

As $\sum_{i=0}^{n} v_{i}=0$, we have that

$$
\begin{aligned}
\pi_{s t} & =\sum_{1 \leq i<j \leq n} v_{i} \wedge v_{j}=\sum_{0 \leq i<j \leq n} v_{i} \wedge v_{j} \\
& =\sum_{0 \leq i<j \leq n-1} v_{i} \wedge v_{j} \\
& =\sigma_{*}^{-1}\left(\pi_{s t}\right),
\end{aligned}
$$

where the last step holds since that

$$
\sigma_{*}\left(v_{i}\right)=\sigma_{*}\left(z_{i} \frac{\partial}{\partial z_{i}}\right)=z_{i+1} \frac{\partial}{\partial z_{i+1}}=v_{i+1} \quad(0 \leq i \leq n-1) .
$$

Therefore the standard Poisson structure is invariant under the map $\sigma$.

Since $\sigma^{n+1}=$ id, we can define a $\mathbb{Z}_{n+1}$-action on $X=\mathbb{C P}^{n}$, generated by $\sigma$. By the arguments above, $\pi_{s t}$ is invariant under the $\mathbb{Z}_{n+1}$-action.

Corollary 4.11. The standard Poisson structure

$$
\pi_{s t}=\sum_{1 \leq i<j \leq n} v_{i} \wedge v_{j}
$$

on $X=\mathbb{C P}^{n}$ is invariant under the $\mathbb{Z}_{n+1}$-action defined above, which induces a $\mathbb{Z}_{n+1}$-action on the Poisson cohomology groups $H_{\pi_{s t}}^{k}(X)(0 \leq k \leq n)$.

In the cases of $X=\mathbb{C} \mathbf{P}^{2}$ (Proposition 4.7) and $X=\mathbb{C} \mathbf{P}^{3}$ (Proposition 4.8), it is easy to verify the $\mathbb{Z}_{3}$-action and the $\mathbb{Z}_{4}$-action on the Poisson cohomology groups. 


\section{Poisson cohomology of holomorphic toric Poisson structures on $\mathbb{C}^{n}$}

In this section, we will study the Poisson cohomology of $X=\mathbb{C}^{n}$. Our setting is based on Example 2.10 and Example 2.15.

Let $W=\rho\left(N_{\mathbb{C}}\right), W^{k}=\wedge^{k} W$ and $W^{0}=\mathbb{C}$. For any $I \in M$, let

$$
m_{i}=\left\langle I, e_{i}\right\rangle(1 \leq i \leq n)
$$

Suppose that $\left\{m_{i_{1}}, m_{i_{2}}, \ldots, m_{i_{l}} \mid 0 \leq i_{1}<i_{2}<\ldots<i_{l} \leq n\right\}$ is the set of all the elements equal to -1 in $\left\{m_{0}, m_{1}, \ldots, m_{n}\right\}$. Set

$$
|I|=l, \quad \chi^{I}=z_{1}^{m_{1}} \cdots z_{n}^{m_{n}}, \quad \mathcal{V}_{I}=v_{i_{1}} \wedge \ldots \wedge v_{i_{l}} \in W^{l}, \quad \mathcal{E}_{I}=e_{i_{1}} \wedge \ldots \wedge e_{i_{l}} \in \wedge^{l} N .
$$

Any holomorphic toric Poisson structure $\pi$ on $X=\mathbb{C}^{n}$ can be written as

$$
\pi=\sum_{1 \leq i<j \leq n} a_{i j} v_{i} \wedge v_{j}
$$

The corresponding $\Pi=\sum_{1 \leq i<j \leq n} a_{i j} e_{i} \wedge e_{j} \in \wedge^{2} N_{\mathbb{C}}$ satisfies $\rho(\Pi)=\pi$. Notice that

$$
\pi=\sum_{1 \leq i<j \leq n} a_{i j} v_{i} \wedge v_{j}=\sum_{1 \leq i<j \leq n} a_{i j} z_{i} z_{j} \frac{\partial}{\partial z_{i}} \wedge \frac{\partial}{\partial z_{j}}
$$

which is called a diagonal quadratic Poisson structure in [21].

For $X=\mathbb{C}^{n}$, since $H^{i}\left(X, \wedge^{j} T X\right)=0(i>0)$, the Poisson cohomology $H_{\pi}^{\bullet}(X)$ is isomorphic to the cohomology of the complex

$$
H^{0}\left(X, \mathcal{O}_{X}\right) \stackrel{d_{\pi}}{\longrightarrow} H^{0}\left(X, T_{X}\right) \stackrel{d_{\pi}}{\longrightarrow} H^{0}\left(X, \wedge^{2} T_{X}\right) \stackrel{d_{\pi}}{\longrightarrow} \ldots \stackrel{d_{\pi}}{\longrightarrow} H^{0}\left(X, \wedge^{n} T_{X}\right),
$$

where $d_{\pi}=[\pi, \cdot]$.

The complex vector space $H^{0}\left(X, \wedge^{k} T X\right)(0 \leq k \leq n)$ is infinite dimensional. Any holomorphic $k$-vector field on $X$ can be written as

$$
\sum_{1 \leq i_{1}<i_{2}<\ldots<i_{k} \leq n} f_{i_{1}, i_{2}, \ldots, i_{k}} \frac{\partial}{\partial z_{i_{1}}} \wedge \ldots \wedge \frac{\partial}{\partial z_{i_{k}}}
$$

where $f_{i_{1}, i_{2}, \ldots, i_{k}}$ are analytic functions on $\mathbb{C}^{n}$ with variables $z_{1}, z_{2}, \ldots, z_{n}$. Analytic functions on $\mathbb{C}^{n}$ can be written as

$$
\sum_{m_{i} \geq 0} a_{m_{1}, \ldots, m_{n}} z_{1}^{m_{1}} \ldots z_{n}^{m_{n}}
$$

satisfying the convergence conditions, which makes the computation of Poisson cohomology difficult.

However, we can consider the algebraic Poisson coholomogy groups and the formal Poisson cohomology groups, which are easier to compute than the Poisson cohomology of holomorphic toric Poisson structures on $\mathbb{C}^{n}$.

- In [21, Monnier considered the formal $k$-vector fields on $R^{n}$ and computed the formal Poisson cohomology of diagonalizable quadratic Poisson structures. His results also applies for $X=\mathbb{C}^{n}$. A formal $k$-vector field on $\mathbb{C}^{n}$ is in the following form

$$
\sum_{1 \leq i_{1}<i_{2}<\ldots<i_{k} \leq n} f_{i_{1}, i_{2}, \ldots, i_{k}} \frac{\partial}{\partial z_{i_{1}}} \wedge \ldots \wedge \frac{\partial}{\partial z_{i_{k}}},
$$


where $f_{i_{1}, i_{2}, \ldots, i_{k}} \in \mathbb{C}\left[\left[z_{1}, z_{2}, \ldots, z_{n}\right]\right]$. We denote $\mathcal{F} H^{0}\left(X, \wedge^{k} T X\right)(0 \leq k \leq n)$ as the complex vector space of all formal $k$-vector fields on $X=\mathbb{C}^{n}$. Then $H^{0}\left(X, \wedge^{k} T X\right)$ can be considered as a subspace of $\mathcal{F} H^{0}\left(X, \wedge^{k} T X\right)$.

The formal Poisson cohomology group $\mathcal{F} H_{\pi}^{\bullet}(X)$ is defined to be the cohomology of the following complex

$$
\mathcal{F} H^{0}\left(X, \mathcal{O}_{X}\right) \stackrel{d_{\pi}}{\longrightarrow} \mathcal{F} H^{0}\left(X, T_{X}\right) \stackrel{d_{\pi}}{\longrightarrow} \mathcal{F} H^{0}\left(X, \wedge^{2} T_{X}\right) \stackrel{d_{\pi}}{\longrightarrow} \ldots \stackrel{d_{\pi}}{\longrightarrow} \mathcal{F} H^{0}\left(X, \wedge^{n} T_{X}\right),
$$

where $d_{\pi}=[\pi, \cdot]$. The formal Poisson cohomology group $\mathcal{F} H_{\pi}^{\bullet}(X)$ can also be considered as the Poisson cohomology group of the Poisson algebra $\mathbb{C}\left[\left[z_{1}, z_{2}, \ldots, z_{n}\right]\right]$ on $X=\mathbb{C}^{n}$, with the Poisson bracket defined by $\pi$.

- In 20, McMillan studied the Poisson algebra of the coordinate ring of an affine Poisson variety. Here we only focus on the case of $X=\mathbb{C}^{n}$. For an algebraic $k$-vector field on $\mathbb{C}^{n}$, we means a $k$-vector field with the following form

$$
\sum_{1 \leq i_{1}<i_{2}<\ldots<i_{k} \leq n} f_{i_{1}, i_{2}, \ldots, i_{k}} \frac{\partial}{\partial z_{i_{1}}} \wedge \ldots \wedge \frac{\partial}{\partial z_{i_{k}}},
$$

where $f_{i_{1}, i_{2}, \ldots, i_{k}} \in \mathbb{C}\left[z_{1}, z_{2}, \ldots, z_{n}\right]$. We denote $\mathcal{A} H^{0}\left(X, \wedge{ }^{k} T X\right)(0 \leq k \leq n)$ as the complex vector space of all algebraic $k$-vector fields on $X=\mathbb{C}^{n}$. Then $\mathcal{A} H^{0}\left(X, \wedge^{k} T X\right)$ can be considered as a subspace of $H^{0}\left(X, \wedge^{k} T X\right)$.

The algebraic Poisson cohomology group $\mathcal{A} H_{\pi}^{\bullet}(X)$ is defined to be the cohomology of the following complex

$$
\mathcal{A} H^{0}\left(X, \mathcal{O}_{X}\right) \stackrel{d_{\pi}}{\longrightarrow} \mathcal{A} H^{0}\left(X, T_{X}\right) \stackrel{d_{\pi}}{\longrightarrow} \mathcal{A} H^{0}\left(X, \wedge^{2} T_{X}\right) \stackrel{d_{\pi}}{\longrightarrow} \ldots \stackrel{d_{\pi}}{\longrightarrow} \mathcal{A} H^{0}\left(X, \wedge^{n} T_{X}\right),
$$

where $d_{\pi}=[\pi, \cdot]$. The algebraic Poisson cohomology group $\mathcal{A} H_{\pi}^{\bullet}(X)$ can also be considered as the Poisson cohomology group of the Poisson algebra $\mathbb{C}\left[z_{1}, z_{2}, \ldots, z_{n}\right]$ on $X=\mathbb{C}^{n}$, with the Poisson bracket defined by $\pi$.

Lemma 5.1. Let $X=\mathbb{C}^{n}$. Let $V_{I}^{k}=\mathbb{C}\left(\chi^{I} \cdot \mathcal{V}_{I}\right) \wedge W^{k-|I|}$ for $|I| \leq k \leq n$. Then we have

(1) The space of formal $k$-vector fields

$$
\mathcal{F} H^{0}\left(X, \wedge^{k} \mathcal{T}_{X}\right)=\prod_{I \in S_{k}} V_{I}^{k}
$$

for $0 \leq k \leq n$, where $S_{k}$ is the set consisting of all $I \in M$ satisfying the conditions

$$
m_{i} \geq-1 \quad(1 \leq i \leq n)
$$

and

$$
|I| \leq k
$$

(2) The space of algebraic k-vector fields

$$
\mathcal{A} H^{0}\left(X, \wedge{ }^{k} \mathcal{T}_{X}\right)=\bigoplus_{I \in S_{k}} V_{I}^{k}
$$

for $0 \leq k \leq n$, where $S_{k}$ is the set consisting of all $I \in M$ satisfying the conditions

$$
m_{i} \geq-1 \quad(1 \leq i \leq n)
$$

and

$$
|I| \leq k
$$


Theorem 5.2. Let $\pi$ be a holomorphic toric Poisson structure on $X=\mathbb{C}^{n}$. Let $V_{I}^{k}=\mathbb{C}\left(\chi^{I} \cdot \mathcal{V}_{I}\right) \wedge$ $W^{k-|I|}$ for $|I| \leq k \leq n$. Then we have

(1) for $0 \leq k \leq n$, we have

$$
\begin{aligned}
& \mathcal{F} H_{\pi}^{k}(X)=\prod_{I \in S_{k}(\pi)} V_{I}^{k}, \\
& \mathcal{A} H_{\pi}^{k}(X)=\bigoplus_{I \in S_{k}(\pi)} V_{I}^{k},
\end{aligned}
$$

where $S_{k}(\pi)$ is the set consisting of all $I \in M$ satisfying

$$
\begin{aligned}
& m_{i} \geq-1 \quad(0 \leq i \leq n) \\
& |I| \leq k
\end{aligned}
$$

and the equation

$$
\left(\imath_{I} \Pi\right) \wedge \mathcal{E}_{I}=0 .
$$

(2) $\mathcal{F} H_{\pi}^{k}(X)=\mathcal{A} H_{\pi}^{k}(X)=0$ for $k>n$.

Lemma 5.1 and Theorem 5.2 can be proved by similar arguments as we have done for $\mathbb{C P}^{n}$. Here we skip the proof.

Remark 5.3. In Lemma 5.1] and Theorem 5.2, the results for formal $k$-vector fields and formal Poisson cohomology were proved by Monnier in 21. Here we write the results from the viewpoint of toric varieties.

By Theorem 5.2, we have the following corollary.

Corollary 5.4. If $S_{k}(\pi)$ is a finite set, then we have $\mathcal{F} H_{\pi}^{k}(X) \cong \mathcal{A} H_{\pi}^{k}(X)$.

Question: if $S_{k}(\pi)$ is a finite set, do we have $\mathcal{F} H_{\pi}^{k}(X) \cong \mathcal{A} H_{\pi}^{k}(X) \cong H_{\pi}^{k}(X)$ ?

\section{REFERENCES}

[1] M. F. Atiyah, Complex analytic connections in fibre bundles, Trans. Amer. Math. Soc. 85 (1957), 181-207. MR0086359

[2] A. Bondal, Noncommutative deformations and Poisson brackets on projective spaces, Preprint MPI/ 93-67 (1993).

[3] Raoul Bott, Homogeneous vector bundles, Ann. of Math. (2) 66 (1957), 203-248. MR0089473

[4] K. A. Brown, K. R. Goodearl, and M. Yakimov, Poisson structures on affine spaces and flag varieties. I. Matrix affine Poisson space, Adv. Math. 206 (2006), no. 2, 567-629, DOI 10.1016/j.aim.2005.10.004. MR2263715

[5] Arlo Caine, Toric Poisson structures, Mosc. Math. J. 11 (2011), no. 2, 205-229, 406 (English, with English and Russian summaries). MR2859234

[6] Zhuo Chen, Anna Fino, and Yat-Sun Poon, Holomorphic Poisson structure and its cohomology on nilmanifolds, Differential Geom. Appl. 44 (2016), 144-160, DOI 10.1016/j.difgeo.2015.11.006. MR3433981

[7] David Cox, What is a toric variety?, Topics in algebraic geometry and geometric modeling, Contemp. Math., vol. 334, Amer. Math. Soc., Providence, RI, 2003, pp. 203-223, DOI 10.1090/conm/334/05983. MR2039974

[8] William Fulton, Introduction to toric varieties, Annals of Mathematics Studies, vol. 131, Princeton University Press, Princeton, NJ, 1993. The William H. Roever Lectures in Geometry. MR1234037

[9] K. R. Goodearl and M. Yakimov, Poisson structures on affine spaces and flag varieties. II, Trans. Amer. Math. Soc. 361 (2009), no. 11, 5753-5780, DOI 10.1090/S0002-9947-09-04654-6. MR2529913

[10] Ryushi Goto, Deformations of generalized complex and generalized Kähler structures, J. Differential Geom. 84 (2010), no. 3, 525-560. MR2669364 
[11] Marco Gualtieri, Generalized complex geometry, Ann. of Math. (2) 174 (2011), no. 1, 75-123, DOI 10.4007/annals.2011.174.1.3. MR2811595

[12] Nigel Hitchin, Instantons, Poisson structures and generalized Kähler geometry, Comm. Math. Phys. 265 (2006), no. 1, 131-164, DOI 10.1007/s00220-006-1530-y. MR2217300

[13] _ Poisson modules and generalized geometry, Geometry and analysis. No. 1, Adv. Lect. Math. (ALM), vol. 17, Int. Press, Somerville, MA, 2011, pp. 403-417. MR2882431

[14] _ Deformations of holomorphic Poisson manifolds, Mosc. Math. J. 12 (2012), no. 3, 567-591, 669 (English, with English and Russian summaries). MR3024823

[15] Wei Hong and Ping Xu, Poisson cohomology of del Pezzo surfaces, J. Algebra 336 (2011), 378-390, DOI 10.1016/j.jalgebra.2010.12.017. MR2802550

[16] Chunghoon Kim, Deformations of Compact Holomorphic Poisson Manifolds and Algebraic Poisson Schemes, ProQuest LLC, Ann Arbor, MI, 2014. Thesis (Ph.D.)-University of California, Riverside. MR3232289

[17] Camille Laurent-Gengoux, Mathieu Stiénon, and Ping Xu, Holomorphic Poisson manifolds and holomorphic Lie algebroids, Int. Math. Res. Not. IMRN, posted on 2008, Art. ID rnn 088, 46, DOI 10.1093/imrn/rnn088. MR2439547

[18] Jiang-Hua Lu and Victor Mouquin, On the T-leaves of some Poisson structures related to products of flag varieties, Adv. Math. 306 (2017), 1209-1261, DOI 10.1016/j.aim.2016.11.008. MR3581329

[19] Evgeny Mayanskiy, Poisson cohomology of two Fano threefolds, J. Algebra 424 (2015), 21-45, DOI 10.1016/j.jalgebra.2014.08.049. MR3293212

[20] Aaron Fraenkel McMillan, Extensions of Poisson Structures on Singular Hypersurfaces, ArXiv e-prints 1310.6083 (2013).

[21] Philippe Monnier, Formal Poisson cohomology of quadratic Poisson structures, Lett. Math. Phys. 59 (2002), no. 3, 253-267, DOI 10.1023/A:1015513632414. MR1904986

[22] Tadao Oda, Convex bodies and algebraic geometry, Ergebnisse der Mathematik und ihrer Grenzgebiete (3) [Results in Mathematics and Related Areas (3)], vol. 15, Springer-Verlag, Berlin, 1988. An introduction to the theory of toric varieties; Translated from the Japanese. MR922894

[23] A. Polishchuk, Algebraic geometry of Poisson brackets, J. Math. Sci. (New York) 84 (1997), no. 5, 1413-1444, DOI 10.1007/BF02399197. Algebraic geometry, 7. MR1465521

[24] Yat Sun Poon, Holomorphic Poisson structures on principle toric bundles, private communication (2016).

School of Mathematics and Statistics, Wuhan University, China

Mathematics Research Unit, University of Luxembourg, Luxembourg

E-mail address: hong_w@whu.edu.cn 